\title{
Static and dynamic properties of the spinless Falicov-Kimball model
}

\author{
K.W. Becker ${ }^{a}$, S. Sykora ${ }^{a}$, and V. Zlatic ${ }^{a, b}$ \\ ${ }^{a}$ Institut für Theoretische Physik, Technische \\ Universität Dresden, D-01062 Dresden, Germany \\ ${ }^{b}$ Institute of Physics, Bijenicka c. 46, P.O.B 304, 10000 Zagreb, Croatia
}

(Dated: July 6, 2018)

\begin{abstract}
The spinless Falicov-Kimball model is studied by the use of a recently developed projector-based renormalization method (PRM) for many-particle Hamiltonians. The method is used to evaluate static and dynamic quantities of the one-dimensional model at half-filling. To these belong the quasiparticle excitation energy $\tilde{\varepsilon}_{k}$ and the momentum distribution $n_{k}$ of the conduction electrons and spatial correlation functions of the localized electrons. One of the most remarkable results is the appearance of a gap in $\tilde{\varepsilon}_{k}$ at the Fermi level of the order of the Coulomb repulsion $U$, which is accompanied by a smooth behavior for $n_{k}$. The density of states for the conduction electrons and the one-particle spectral functions for the localized electrons are also discussed. In both quantities a gap opens with increasing $U$.
\end{abstract}

PACS numbers: 71.10.Fd, 71.27.+a, 75.30.Mb 


\section{INTRODUCTION}

The Falicov-Kimball model is a widely used model to study the properties of interacting fermions $\frac{1}{2}$ and has also been used to describe the valence change transition in $\mathrm{YbInCu}_{4}$ and similar compounds 2 . (For a recent review see Ref. 3). The model considers a lattice of localized $f$-sites, which are either empty or singly-occupied site, and of conduction electron sites, which are delocalized by the nearest-neighbor hopping. There are $n_{f} f$-electrons and $n_{c}$ conduction electrons interacting by an on-site Coulomb interaction $U$. The two types of electrons share the common chemical potential $\mu$, which is always adjusted so as to keep $n_{f}+n_{c}=1$. The local $f$-charge is a constant of motion but thermal fluctuations can change the average $f$-occupation by transferring electrons or holes from the conduction band to $f$-states. The relative occupation of $f$-states and conduction states is determined by the competition between the entropy of the $f$ - and the conduction states, the excitation energy of the f-states, the kinetic energy of band states, and the interaction energy.

The general solution of the Falicov-Kimball model is not known except in infinite dimension $\underline{\underline{3}, 4, \underline{5}}$. In low dimensions, the zero-temperature phase diagram is highly non-trivial and has attracted a lot of theoretical attention (see (3) for the list of references). In one dimension the thermodynamic properties of the model are more or less understood, whereas dynamical properties have been much less studied. Kennedy and Lieb $\underline{\underline{6}} \underline{7}$ proved that at low enough temperatures the half-filled Falicov-Kimball model for dimensions $d \geq 2$ possesses long-range order, i.e., the ions form a checkerboard pattern, the same as in the ground state. In the ordered phase, the lattice can be divided into two inter-penetrating sublattices $\mathrm{A}$ and $\mathrm{B}$ in such a way that all nearest neighbors of a site from sublattice A belong to sublattice $\mathrm{B}$ and vice versa. This result holds for any value of the Coulomb interaction $U$. For one dimension longe range order exists only at temperature $T=0$.

In what follows, we first describe the Hamiltonian and the projector-based renormalization method (PRM) which was recently introduced (Sect. I). In Sect. II the method is then applied to the one-dimensional Falicov-Kimball model and renormalization equations are derived for the parameters of the Hamiltonian. The results for static and dynamic quantities are discussed in Sect. III and IV.

To simplify the calculation, we consider only the case with translation symmetry. This excludes the discussion of physical properties which depend on lattice sites $i$, i.e. we do 
not discuss the ordered phase which emerges at low temperatures. However, as discussed in Sect. V, we can still evaluate physical quantities which are given by averages over all lattice sites. Note that the PRM approach can also be extended to situations with long range order. This was shown for instance in Ref. (8), where the quantum phase transition for the spinless Holstein model was discussed as function of the electron-phonon coupling.

\section{A. Model}

The spinless Falicov-Kimball model is described by the Hamiltonian

$$
\mathcal{H}=\sum_{<i, j>} t_{i, j} c_{i}^{\dagger} c_{j}+\varepsilon_{f} \sum_{i} n_{i}+U \sum_{i} n_{i} n_{i}^{c},
$$

where, $c_{i}^{\dagger}$ and $c_{i}$ are the creation and annihilation operators for conduction electrons at site $i, t_{i, j}$ are the hopping matrix elements, $\varepsilon_{f}$ is the energy level of the localized electrons,

and $n_{i}=f_{i}^{\dagger} f_{i}$ and $n_{i}^{c}=c_{i}^{\dagger} c_{i}$ are the occupation number operators of the localized and conduction electrons at site $i$. The summation runs over the $N$ sites of a periodic lattice, and $U$ is the interaction strength of the local Coulomb repulsion at sites $i$ between conduction and localized electrons. Note that $n_{i}$ at each site $i$ commutes with the Hamiltonian. Thus, the $f$-electron occupation number is a good quantum number, taking only two values 1 or 0 , according to whether or not site $i$ is occupied by the localized $f$-electron.

\section{B. Projector-based renormalization method (PRM)}

Let us first discuss a recently introduced many-particle method, the projector-based renormalization method $(\mathrm{PRM})^{\frac{9}{}}$, which provides an approximate solution to many-particle problems defined by the Hamiltonian $\mathcal{H}$,

$$
\mathcal{H}=\mathcal{H}_{0}+\mathcal{H}_{1}
$$

Here, $\mathcal{H}_{0}$ is the unperturbed part with a known eigenvalue spectrum, $\mathcal{H}_{0}|n\rangle=E_{n}^{(0)}|n\rangle$, and $\mathcal{H}_{1}$ is the perturbation which does not commute with $\mathcal{H}_{0}$ and has off-diagonal matrix elements, $\left\langle n\left|\mathcal{H}_{1}\right| m\right\rangle \neq 0$, but no diagonal elements. In a usual perturbative approach one evaluates the corrections to the eigenstates and eigenvalues of $\mathcal{H}_{0}$ due to $\mathcal{H}_{1}$. The n-th order correction requires the evaluation of all matrix elements up to $\left\langle n\left|\left(\mathcal{H}_{1}\right)^{n}\right| m\right\rangle$, which are difficult to calculate. 
An alternative insight is obtained by making a unitary transformation to a new basis which has no transition matrix elements with energy differences larger than some chosen cutoff $\lambda<\Lambda$. Here $\Lambda$ is the largest energy difference between any two eigenstates of $\mathcal{H}_{0}$ which are connected by $\mathcal{H}_{1}$. This generates a new Hamiltonian

$$
\mathcal{H}_{\lambda}=e^{X_{\lambda}} \mathcal{H} e^{-X_{\lambda}}
$$

which can be written as a sum of two terms,

$$
\mathcal{H}_{\lambda}=\mathcal{H}_{0, \lambda}+\mathcal{H}_{1, \lambda}
$$

such that $\left\langle m\left|\mathcal{H}_{1, \lambda}\right| n\right\rangle=0$ for $\left|E_{n}^{\lambda}-E_{m}^{\lambda}\right|>\lambda$, where $E_{n}^{\lambda}$ and $|n\rangle$ are the new eigenvalues and eigenstates of $\mathcal{H}_{0, \lambda} \cdot \mathcal{H}_{1, \lambda}$ is chosen in such a way that it has no diagonal elements with respect to $\mathcal{H}_{0, \lambda}$. The generator of the unitary transformation is denoted by $X_{\lambda}$ which has to be anti-hermitian $X_{\lambda}^{\dagger}=-X_{\lambda}$. Note that the elimination of high energy transitions may also generate new interaction terms which have operator structures different from that of $\mathcal{H}_{1}$. However, they do not connect states with an energy separation larger than $\lambda$.

In the PRM method the elimination of transition matrix elements is carried out by defining a generalized projection operator $\mathbf{P}_{\lambda}$ which removes from any operator $\mathcal{A}$ those parts which give rise to 'forbidden' transitions, i.e.,

$$
\mathbf{P}_{\lambda} \mathcal{A}=\sum_{\substack{m, n \\\left|E_{n}^{\lambda}-E_{m}^{\lambda}\right| \leq \lambda}}|n\rangle\langle m|\langle n|\mathcal{A}| m\rangle
$$

i.e. we retain in (5) only the states $|m\rangle$ and $|n\rangle$ with $\left|E_{n}^{\lambda}-E_{m}^{\lambda}\right| \leq \lambda$. The orthogonal complement of $\mathbf{P}_{\lambda}$ is $\mathbf{Q}_{\lambda}=\mathbf{1}-\mathbf{P}_{\lambda}$. Here only dyads $|n\rangle\langle m|$ contribute with $\left|E_{n}-E_{m}\right|>\lambda$.

The generator $X_{\lambda}$ in (3) is determined by the condition

$$
\mathrm{Q}_{\lambda} \mathcal{H}_{\lambda}=0
$$

as can be seen by expanding the exponentials in (3). This gives

$$
\mathrm{Q}_{\lambda}\left\{\mathcal{H}_{1}+\sum_{n=1}^{\infty} \frac{1}{n !} \mathbf{X}_{\lambda}{ }^{n} \mathcal{H}\right\}=0
$$

where $\mathbf{X}_{\lambda}$ is a superoperator defined by $\mathbf{X}_{\lambda} \mathcal{H}=\left[X_{\lambda}, \mathcal{H}\right], \mathbf{X}_{\lambda}{ }^{2} \mathcal{H}=\left[X_{\lambda},\left[X_{\lambda}, \mathcal{H}\right]\right]$, etc. In lowest order, $\mathbf{Q}_{\lambda} \mathcal{H}_{\lambda}=0$ reduces to $\mathbf{Q}_{\lambda}\left\{\mathcal{H}_{1}-\mathbf{L}_{0} X_{\lambda}^{(1)}\right\}=0$, where $\mathbf{L}_{0} \mathcal{A}=\left[\mathcal{H}_{0}, \mathcal{A}\right]$ defines 
the unperturbed Liouville operator $\mathbf{L}_{0}$, which commutes with $\mathbf{Q}_{\lambda}$. Obviously, the condition $\mathbf{Q}_{\lambda} \mathcal{H}_{\lambda}=0$ is satisfied by the expression $X_{\lambda}^{(1)}=\mathbf{L}_{0}^{-1} \mathbf{Q}_{\lambda} \mathcal{H}_{1}$, i.e., to lowest order, $X_{\lambda}^{(1)}$ can be obtained from the decomposition of $\mathcal{H}_{1}$ into the eigenmodes of $\mathbf{L}_{0}$. The higher order correction terms to $X_{\lambda}$ follow systematically from the higher order commutators of (7) (for details see, e.g., Ref. 9).

We see from Eq. (17) that all transitions in $\mathcal{H}_{1}$ with energy transfers between the original cutoff $\Lambda$ and the new cutoff $\lambda$ are eliminated in one step. However it is more convenient to perform the elimination procedure step-wise, such that each step reduces the cutoff energy $\lambda$ by a small amount $\Delta \lambda$. Thus, the first step removes all the transitions which involve energy transfers between (the original cutoff) $\Lambda$ and $\Lambda-\Delta \lambda$, the subsequent steps remove all transitions larger than $\Lambda-2 \Delta \lambda, \Lambda-3 \Delta \lambda$, and so on. The unitary transformation for the step from an intermediate cutoff $\lambda$ to the new cutoff $\lambda-\Delta \lambda$ reads (in analogy to (3))

$$
\mathcal{H}_{\lambda-\Delta \lambda}=e^{X_{\lambda, \Delta \lambda}} \mathcal{H}_{\lambda} e^{-X_{\lambda, \Delta \lambda}}
$$

where $\mathcal{H}_{\lambda}=\mathcal{H}_{0, \lambda}+\mathcal{H}_{1, \lambda}$ and $\mathcal{H}_{\lambda-\Delta \lambda}=\mathcal{H}_{0, \lambda-\Delta \lambda}+\mathcal{H}_{1, \lambda-\Delta \lambda}$. The generator $X_{\lambda, \Delta \lambda}$ is now fixed by the condition

$$
\mathrm{Q}_{\lambda-\Delta \lambda} \mathcal{H}_{\lambda-\Delta \lambda}=0
$$

which specifies that $\mathcal{H}_{\lambda-\Delta \lambda}$ contains no matrix elements which connect eigenstates of $\mathcal{H}_{0, \lambda-\Delta \lambda}$ with energy differences larger than $\lambda-\Delta \lambda$.

Let us assume that the operator structure of $\mathcal{H}_{\lambda}$ is invariant with respect to further unitary transformations. Then, we can use Eq. (17) to derive difference equations for the $\lambda$-dependence of the coupling constants of the Hamiltonian. These equations connect the parameters of the Hamiltonian with cutoff $\lambda$ to those with cutoff $\lambda-\Delta \lambda$. By using a finite number of steps we can proceed to $\lambda \rightarrow 0$ and obtain a set of nonlinear equations for the renormalized parameters. The solution determines the final, fully renormalized Hamiltonian $\mathcal{H}_{\lambda \rightarrow 0}=\mathcal{H}_{0, \lambda \rightarrow 0}$, which depends on the initial parameter values of the original model at cutoff $\Lambda$. In this limit all transitions due to the interaction with nonzero transition energies have been eliminated so that $\mathcal{H}_{1, \lambda \rightarrow 0}$ identically vanishes, i.e. $\mathcal{H}_{1, \lambda \rightarrow 0}=0$.

The underlying idea of the PRM, namely the elimination of the interaction terms by the unitary transformations, has been used before in the literature. For instance, in Ref.10 a unitary transformation has been employed to eliminate off-diagonal matrix elements of 
the Coulomb interaction in order to study ground state properties of the Hubbard model. Similarly, in the Schrieffer-Wolff transformation ${ }^{11}$, which maps the Anderson to the Kondo model, matrix elements connecting different charge configurations of magnetic ions are eliminated also by use of a unitary transformation. Note however that in the PRM approach unitary transformations are performed in small steps in contrast to earlier applications. Note also that the approach removes high energy transitions but does not decimate the Hilbert space. This is different to the poor man's scaling $\frac{12}{}$ which removes high energy states. It should also be mentioned that the PRM resembles the previous flow equation method ${ }^{13}$ by Wegner and the similarity renormalization approach ${ }^{14}$ by Glazek and Wilson which can be considered as continuous versions of the PRM method.

\section{RENORMALIZATION OF THE FALICOV-KIMBALL MODEL}

In order to apply the PRM we express the Falicov-Kimball interaction in terms of the fluctuations with respect to the thermal averages of $n_{i}^{c}$ and $n_{i}$, and write the diagonal and off-diagonal part of $\mathcal{H}$ as

$$
\mathcal{H}_{0}=\sum_{i}\left(\varepsilon_{f}+U\left\langle n_{i}^{c}\right\rangle\right) n_{i}+\sum_{k}\left(\varepsilon_{k}+U\left\langle n_{i}\right\rangle\right) c_{k}^{\dagger} c_{k}-U N\left\langle n_{i}\right\rangle\left\langle n_{i}^{c}\right\rangle
$$

and

$$
\mathcal{H}_{1}=\frac{U}{N} \sum_{i k k^{\prime}} e^{-i\left(k-k^{\prime}\right) R_{i}} \delta n_{i} \delta\left(c_{k}^{\dagger} c_{k^{\prime}}\right)
$$

where $\delta n_{i}^{c}=n_{i}^{c}-\left\langle n_{i}^{c}\right\rangle, \delta n_{i}=n_{i}-\left\langle n_{i}\right\rangle,\langle\cdots\rangle$ denotes the thermal averaging with the full Hamiltonian $\mathcal{H}$, and $c_{k}^{\dagger}=(1 / \sqrt{N}) \sum_{i} e^{i k R_{i}} c_{i}^{\dagger}$ is the Fourier transform of $c_{i}^{\dagger}$. Of course, due to the assumed translation invariance $\left\langle n_{i}\right\rangle$ is equivalent to $(1 / N) \sum_{i}\left\langle n_{i}\right\rangle$, where $N$ is the number of lattice sites. The sum in (11) is restricted to $k \neq k^{\prime}$, since the diagonal part of the Coulomb repulsion is included in $\mathcal{H}_{0}$. From translation invariance it follows $\delta\left(c_{k}^{\dagger} c_{k^{\prime}}\right)=c_{k}^{\dagger} c_{k^{\prime}}-\left\langle c_{k}^{\dagger} c_{k}\right\rangle \delta_{k, k^{\prime}}$. In principle, the average values in (10) can be defined with an arbitrary ensemble since Eq. (1) and Eqs. (10), (11) are equivalent. It has turned out that the best choice is to evaluate the averages with respect to the original full Hamiltonian $\mathcal{H}=\mathcal{H}_{\Lambda}$, which can be done by the procedure explained below.

The perturbation $\mathcal{H}_{1}$ causes transitions between the eigenstates of $\mathcal{H}_{0}$ by creating electron-hole pairs in the conduction band, whereas the number of $f$-electrons is conserved 
at each site. Using $\mathbf{L}_{0} \delta n_{i} \delta\left(c_{k}^{\dagger} c_{k^{\prime}}\right)=\left(\varepsilon_{k}-\varepsilon_{k^{\prime}}\right) \delta n_{i} \delta\left(c_{k}^{\dagger} c_{k^{\prime}}\right), \mathcal{H}_{1}$ can be decomposed into a sum of eigenmodes of the unperturbed Liouville operator. The lowest order solution of the generator of the unitary transformation is $X_{\lambda}^{(1)}=\mathbf{L}_{0}^{-1} \mathbf{Q}_{\lambda} \mathcal{H}_{1}$, which is given by

$$
X_{\lambda}^{(1)}=\frac{U}{N} \sum_{i k k^{\prime}} \frac{e^{i\left(k-k^{\prime}\right) R_{i}}}{\varepsilon_{k}-\varepsilon_{k^{\prime}}} \Theta\left(\left|\varepsilon_{k}-\varepsilon_{k^{\prime}}\right|-\lambda\right) \delta n_{i} c_{k}^{\dagger} c_{k^{\prime}}
$$

Here $\Theta\left(\left|\varepsilon_{k}-\varepsilon_{k^{\prime}}\right|-\lambda\right)$ is the projection operator which removes from $\mathcal{H}_{1}$ all transitions with energy transfers larger than $\lambda$. Note that the same operator form also appears in the non-perturbative calculation below.

\section{A. Renormalized Hamiltonian}

The unitary transformation leads to the renormalized Hamiltonian $\mathcal{H}_{\lambda}=e^{X_{\lambda}} \mathcal{H} e^{-X_{\lambda}}$, which can be written as $\mathcal{H}_{\lambda}=\mathcal{H}_{0, \lambda}+\mathcal{H}_{1, \lambda}$, where $\mathcal{H}_{1, \lambda}$ gives rise to the transitions between the eigenstates of $\mathcal{H}_{0, \lambda}$ but has no diagonal elements. The choice of $\mathcal{H}_{0, \lambda}$ is not unique, and each particular case requires a physical intuition. But in any case it has to be such that the thermal averages which appear in the procedure can be evaluated. For the Falicov-Kimball model, the choice which emphasizes the weak coupling limit, is

$$
\begin{aligned}
& \mathcal{H}_{0, \lambda}=\varepsilon_{f, \lambda} \sum_{i} \delta n_{i}+\sum_{i \neq j} g_{i j, \lambda} \delta n_{i} \delta n_{j}+\sum_{k} \varepsilon_{k, \lambda} c_{k}^{\dagger} c_{k}+E_{\lambda}, \\
& \mathcal{H}_{1, \lambda}=\frac{U}{N} \sum_{i k k^{\prime}} \Theta\left(\lambda-\left|\varepsilon_{k, \lambda}-\varepsilon_{k^{\prime}, \lambda}\right|\right) e^{i\left(k-k^{\prime}\right) R_{i}}\left(\delta n_{i} \delta\left(c_{k}^{\dagger} c_{k^{\prime}}\right)\right),
\end{aligned}
$$

where $\varepsilon_{f, \lambda}, g_{i j, \lambda}$, and $\varepsilon_{k, \lambda}$ are the renormalized parameters which are calculated below. Note that the interaction parameter $U$ will not change in the renormalization procedure below so that a $\lambda$-dependence of $U$ is not considered from the beginning. The second term in (13) is a new density-density interaction between the localized electrons which is generated during the renormalization procedure. Additional higher order operator terms, which are also generated by the renormalization, are neglected. The original model $(\lambda=\Lambda)$ is defined by the initial condition, according to (10), (11)

$$
\begin{array}{ll}
\varepsilon_{f, \Lambda}=\varepsilon_{f}+U\left\langle n_{i}^{c}\right\rangle & \varepsilon_{k, \Lambda}=\varepsilon_{k}+U\left\langle n_{i}\right\rangle \\
g_{i j, \Lambda}=0 & E_{\Lambda}=-N\left\langle n_{i}\right\rangle\left(\varepsilon_{f}+U\left\langle n_{i}^{c}\right\rangle\right) .
\end{array}
$$


Once we have made the ansatz (13) and (14) for the operator structure of $\mathcal{H}_{0, \lambda}$ and $\mathcal{H}_{1, \lambda}$, we reduce the cutoff to $\lambda-\Delta \lambda$ to find the new effective Hamiltonian

$$
\mathcal{H}_{\lambda-\Delta \lambda}=e^{X_{\lambda, \Delta \lambda}} \mathcal{H}_{\lambda} e^{-X_{\lambda, \Delta \lambda}}=\mathcal{H}_{0, \lambda-\Delta \lambda}+\mathcal{H}_{1, \lambda-\Delta \lambda}
$$

with renormalized parameters. The new Hamiltonian is such that $\mathcal{H}_{1, \lambda-\Delta \lambda}$ does not give rise to transitions with energy transfers larger than $(\lambda-\Delta \lambda)$ (with respect to the new unperturbed part $\left.\mathcal{H}_{0, \lambda-\Delta \lambda}\right) . \mathcal{H}_{\lambda-\Delta \lambda}$ should have the same operator structure as $\mathcal{H}_{\lambda}$, however with renormalized parameters

$$
\begin{aligned}
\mathcal{H}_{\lambda-\Delta \lambda} & =\varepsilon_{f,(\lambda-\Delta \lambda)} \sum_{i} \delta n_{i}+\sum_{i \neq j} g_{i j,(\lambda-\Delta \lambda)} \delta n_{i} \delta n_{j}+\sum_{k} \varepsilon_{k,(\lambda-\Delta \lambda)} c_{k}^{\dagger} c_{k}+E_{(\lambda-\Delta \lambda)} \\
& +\frac{U}{N} \sum_{i k k^{\prime}} \Theta\left(\lambda-\Delta \lambda-\left|\varepsilon_{k, \lambda-\Delta \lambda}-\varepsilon_{k^{\prime}, \lambda-\Delta \lambda}\right|\right) e^{i\left(k-k^{\prime}\right) R_{i}}\left(\delta n_{i} \delta\left(c_{k}^{\dagger} c_{k^{\prime}}\right)\right) .
\end{aligned}
$$

\section{B. Unitary transformation}

For the explicit evaluation of the unitary transformation (16) we need the generator $X_{\lambda, \Delta \lambda}$. We make the following ansatz

$$
X_{\lambda, \Delta \lambda}=\frac{1}{N} \sum_{k, k^{\prime}, i} A_{k, k^{\prime}}^{\lambda, \Delta \lambda} \Theta_{k k^{\prime}}^{\lambda, \Delta \lambda} e^{-i\left(k-k^{\prime}\right) R_{i}} \delta n_{i} \delta\left(c_{k}^{\dagger} c_{k^{\prime}}\right) .
$$

Here $\Theta_{k k^{\prime}}^{\lambda, \Delta \lambda}$ is a product of $\Theta$-functions

$$
\Theta_{k k^{\prime}}^{\lambda, \Delta \lambda}=\Theta\left(\lambda-\left|\varepsilon_{k, \lambda}-\varepsilon_{k^{\prime}, \lambda}\right|\right) \times \Theta\left[\left|\varepsilon_{k,(\lambda-\Delta \lambda)}-\varepsilon_{k^{\prime},(\lambda-\Delta \lambda)}\right|-(\lambda-\Delta \lambda)\right],
$$

which project all the operators in $X_{\lambda, \Delta \lambda}$ 'on the shell' between $\lambda$ and $\lambda-\Delta \lambda$. More precisely, the energy differences $\left|\varepsilon_{k, \lambda}-\varepsilon_{k^{\prime}, \lambda}\right|$ and $\left|\varepsilon_{k,(\lambda-\Delta \lambda)}-\varepsilon_{k^{\prime},(\lambda-\Delta \lambda)}\right|$ in (19) refer to the two different Hamiltonians $\mathcal{H}_{\lambda}$ and $\mathcal{H}_{\lambda-\Delta \lambda}$. Therefore, the two $\Theta$-functions in (19) take into account that $\mathcal{H}_{\lambda}$ possesses only transition elements with energy transfer $\left|\varepsilon_{k, \lambda}-\varepsilon_{k^{\prime}, \lambda}\right|<\lambda$, whereas in $\mathcal{H}_{\lambda-\Delta \lambda}$ no transitions with $\left|\varepsilon_{k,(\lambda-\Delta \lambda)}-\varepsilon_{k^{\prime},(\lambda-\Delta \lambda)}\right|>\lambda-\Delta \lambda$ are allowed. The coefficients $A_{k, k^{\prime}}^{\lambda, \Delta \lambda}$ are determined below, using the condition $\mathrm{Q}_{\lambda-\Delta \lambda} \mathcal{H}_{\lambda-\Delta \lambda}=0$. Again, the form of $X_{\lambda, \Delta \lambda}$ is suggested by its lowest order expression according to (12) (compare (9)). The unitarity

of the transformation requires $X_{\lambda, \Delta \lambda}=-X_{\lambda, \Delta \lambda}^{\dagger}$, so that $A_{k, k^{\prime}}^{\lambda, \Delta \lambda}$ has to be antisymmetric with respect to the interchange of $k$ and $k^{\prime}$. Expanding the exponentials in (16) the transformation can be written as,

$$
\mathcal{H}_{\lambda-\Delta \lambda}=e^{X_{\lambda, \Delta \lambda}} \mathcal{H}_{\lambda} e^{-X_{\lambda, \Delta \lambda}}=\mathcal{H}_{\lambda}+\sum_{n=1}^{\infty} \frac{1}{n !} \mathbf{X}_{\lambda, \Delta \lambda}^{n} \mathcal{H}_{\lambda}
$$


where $\mathbf{X}_{\lambda, \Delta \lambda} \mathcal{H}_{\lambda}=\left[X_{\lambda, \Delta \lambda}, \mathcal{H}_{\lambda}\right], \mathbf{X}_{\lambda, \Delta \lambda}^{2} \mathcal{H}_{\lambda}=\left[X_{\lambda, \Delta \lambda},\left[X_{\lambda, \Delta \lambda}, \mathcal{H}_{\lambda}\right]\right]$, etc. The commutators are evaluated in Appendix A. The basic approximation of the PRM approach is to replace some of the operators generated by $X_{\lambda, \Delta \lambda}$ by ensemble averages, so as to keep the structure of $\mathcal{H}_{\lambda}$ invariant during the renormalization procedure. This additional factorization enables us to resum the series in (201).

\section{Renormalization of the coupling constants}

By comparing the respective operators in (17) with those obtained from (20) one finds renormalization equations for the parameters of $\mathcal{H}_{\lambda}$. First, from the prefactors of $\delta n_{i}$ one obtains

$$
\begin{aligned}
\varepsilon_{f,(\lambda-\Delta \lambda)} & =\varepsilon_{f, \lambda}+\frac{\left(1-2\left\langle n_{i}\right\rangle\right)}{2 N} \sum_{k k^{\prime}} \Theta_{k, k^{\prime}}^{\lambda, \Delta \lambda}\left(\varepsilon_{k^{\prime}, \lambda}-\varepsilon_{k, \lambda}\right) \frac{\left(1-\cos v_{k k^{\prime}}^{\lambda}\right)}{C_{\rho}\left(k-k^{\prime}\right)}\left\langle c_{k}^{\dagger} c_{k}\right\rangle \\
& +\frac{U\left(1-2\left\langle n_{i}\right\rangle\right)}{N \sqrt{N}} \sum_{k k^{\prime}} \Theta_{k, k^{\prime}}^{\lambda, \Delta \lambda} \frac{\sin v_{k k^{\prime}}^{\lambda}}{\sqrt{C_{\rho}\left(k-k^{\prime}\right)}}\left\langle c_{k}^{\dagger} c_{k}\right\rangle .
\end{aligned}
$$

Similarly, by comparing the coefficients of $c_{k}^{\dagger} c_{k}$ one finds

$$
\begin{aligned}
\varepsilon_{k,(\lambda-\Delta \lambda)} & =\varepsilon_{k, \lambda}+\frac{1}{2} \sum_{k^{\prime}} \Theta_{k, k^{\prime}}^{\lambda, \Delta \lambda}\left(\varepsilon_{k^{\prime}, \lambda}-\varepsilon_{k, \lambda}\right)\left(1-\cos v_{k k^{\prime}}^{\lambda}\right) \\
& +\frac{U}{\sqrt{N}} \sum_{k^{\prime}} \Theta_{k, k^{\prime}}^{\lambda, \Delta \lambda} \sqrt{C_{\rho}\left(k-k^{\prime}\right)} \sin v_{k k^{\prime}}^{\lambda} .
\end{aligned}
$$

The renormalization equation for the interaction parameter $g_{i j, \lambda}$ between $f$-electrons is best expressed in terms of its Fourier transform $g_{q, \lambda}$,

$$
\begin{aligned}
g_{q,(\lambda-\Delta \lambda)} & =g_{q, \lambda}+\frac{1}{4} \sum_{k} \frac{\left\langle c_{k}^{\dagger} c_{k}\right\rangle-\left\langle c_{k+q}^{\dagger} c_{k+q}\right\rangle}{C_{\rho}(q)} \Theta_{k, k+q}^{\lambda, \Delta \lambda}\left(\varepsilon_{k+q, \lambda}-\varepsilon_{k, \lambda}\right)\left(1-\cos v_{k, k+q}^{\lambda}\right) \\
& +\frac{U}{2 \sqrt{N}} \sum_{k} \frac{\left\langle c_{k}^{\dagger} c_{k}\right\rangle-\left\langle c_{k+q}^{\dagger} c_{k+q}\right\rangle}{\sqrt{C_{\rho}(q)}} \Theta_{k, k+q}^{\lambda, \Delta \lambda} \sin v_{k, k+q}^{\lambda} \\
& -\frac{1}{N} \sum_{q^{\prime}}(\cdots)
\end{aligned}
$$

where $(\cdots)$ denotes the second and third term on the r.h.s. with the wave vector $q$ replaced by $q^{\prime}$. In this way, the exact sum rule $\sum_{q} g_{q, \lambda}=0$ is fulfilled which guarantees that only sites with $i \neq j$ contribute to $g_{i j}$. Finally, also the renormalization equation for the energy 
shift $E_{\lambda}$ can be obtained which will not be given explicitly. The other new quantities in Eqs. (21) to (23) are defined as follows

$$
v_{k k^{\prime}}^{\lambda}=2 \sqrt{\frac{C_{\rho}\left(k-k^{\prime}\right)}{N}} A_{k, k^{\prime}}^{\lambda, \Delta \lambda}, \quad C_{\rho}(q)=\frac{1}{N} \sum_{i j} e^{i q\left(R_{i}-R_{j}\right)}\left\langle\delta n_{i} \delta n_{j}\right\rangle .
$$

Finally we have to determine the coefficients $A_{k, k^{\prime}}^{\lambda, \Delta \lambda}$ of the unitary transformation. They follow from the condition $\mathbf{Q}_{\lambda-\Delta \lambda} \mathcal{H}_{\lambda-\Delta \lambda}=0$, which removes from $\mathcal{H}_{\lambda-\Delta \lambda}$ all the operators giving rise to the transitions with energy transfers larger than $\lambda-\Delta \lambda$. One finds

$$
\Theta_{k k^{\prime}}^{\lambda, \Delta \lambda} A_{k, k^{\prime}}^{\lambda, \Delta \lambda}=\Theta_{k k^{\prime}}^{\lambda, \Delta \lambda} \frac{1}{2} \sqrt{\frac{N}{C_{\rho}\left(k-k^{\prime}\right)}} \arctan \left(2 \sqrt{\frac{C_{\rho}\left(k-k^{\prime}\right)}{N}} \frac{U}{\varepsilon_{k, \lambda}-\varepsilon_{k^{\prime}, \lambda}}\right) .
$$

Note that the $A_{k, k^{\prime}}^{\lambda, \Delta \lambda}$ depend on the parameters at cutoff $\lambda$ as well as from the reduced cutoff $\lambda-\Delta \lambda$. The presence of $\Theta_{k k^{\prime}}^{\lambda, \Delta \lambda}$ on both sides of Eq.(25) means that $A_{k, k^{\prime}}^{\lambda, \Delta \lambda}$ has to be specified only for those values of $k$ and $k^{\prime}$ which are 'on the shell' defined by the condition $\Theta_{k k^{\prime}}^{\lambda, \Delta \lambda}=1$. 'Off-the-shell' coefficients can take any value and, in what follows, we use $A_{k, k^{\prime}}^{\lambda, \Delta \lambda}=0$ for $\Theta_{k k^{\prime}}^{\lambda, \Delta \lambda}=0$. Note that expression (25) includes $U$ to all orders. However, in the thermodynamic limit $N \rightarrow \infty$, the coefficients $A_{k, k^{\prime}}^{\lambda, \Delta \lambda}$ become linear in $U$ since all higher order terms vanish for $N \rightarrow \infty$. In the actual numerical evaluation of the renormalization equations on a lattice of finite size $N$ the excitation energies $\left(\varepsilon_{k^{\prime}, \lambda}-\varepsilon_{k, \lambda}\right)$ may become very small for $\lambda \rightarrow 0$ so that the expansion of $A_{k, k^{\prime}}^{\lambda, \Delta \lambda}$ to linear order in $U$ breaks down. In this case, the full expression (25) has to be taken.

By help of (25) one finds for the parameters $v_{k k^{\prime}}^{\lambda}$ of (24)

$$
\begin{aligned}
\sin v_{k k^{\prime}}^{\lambda} & =2 U \sqrt{\frac{C_{\rho}\left(k-k^{\prime}\right)}{N}} \frac{\operatorname{sign}\left(\varepsilon_{k, \lambda}-\varepsilon_{k^{\prime}, \lambda}\right)}{\sqrt{\left(\varepsilon_{k, \lambda}-\varepsilon_{k^{\prime}, \lambda}\right)^{2}+4 U^{2} C_{\rho}\left(k-k^{\prime}\right) / N}} \\
\cos v_{k k^{\prime}}^{\lambda} & =\frac{\left|\varepsilon_{k, \lambda}-\varepsilon_{k^{\prime}, \lambda}\right|}{\sqrt{\left(\varepsilon_{k, \lambda}-\varepsilon_{k^{\prime}, \lambda}\right)^{2}+4 U^{2} C_{\rho}\left(k-k^{\prime}\right) / N}} \cdot
\end{aligned}
$$

Equations (21) to (23) represent the final renormalization equations for the parameters of the Hamiltonian as the energy cutoff is reduced from $\lambda$ to $\lambda-\Delta \lambda$. The overall renormalization starts from the original cutoff $\lambda=\Lambda$, where the initial parameters are given by (15). For the parameters $\sin v_{k k^{\prime}}^{\lambda}, \cos v_{k k^{\prime}}^{\lambda}$, and $C_{\rho}\left(k-k^{\prime}\right)$, which contain expectation values, an appropriate choice is taken at cutoff $\Lambda$. Using these quantities we obtain from the renormalization 
equations (21) to (23) the renormalized parameters at the smaller cutoff $\Lambda-\Delta \lambda$. The procedure is repeated until the cutoff is reduced to zero. At $\lambda=0$, we obtain the completely renormalized Hamiltonian $\tilde{\mathcal{H}}:=\mathcal{H}_{(\lambda=0)}$, which describes an effectively decoupled system of free conduction electrons and interacting localized electrons,

$$
\tilde{\mathcal{H}}=\tilde{\mathcal{H}}_{c}+\tilde{\mathcal{H}}_{f}+\tilde{E}
$$

where

$$
\tilde{\mathcal{H}}_{c}=\sum_{k} \tilde{\varepsilon}_{k} c_{k}^{\dagger} c_{k}, \quad \text { and } \quad \tilde{\mathcal{H}}_{f}=\tilde{\varepsilon}_{f} \sum_{i} \delta n_{i}+\sum_{i \neq j} \tilde{g}_{i j} \delta n_{i} \delta n_{j}
$$

The fully renormalized parameters are defined as $\tilde{\varepsilon}_{k}=\varepsilon_{k,(\lambda=0)}, \tilde{\varepsilon}_{f}=\varepsilon_{f,(\lambda=0)}, \tilde{g}_{i j}=g_{i j,(\lambda=0)}$ and $\tilde{E}=E_{(\lambda=0)}$. Note that for $\lambda \rightarrow 0$ the interaction $\mathcal{H}_{1, \lambda}$ has completely vanished due to the $\Theta$-function in (14). Using $\tilde{\mathcal{H}}$ we next can recalculate the statistical averages $\left\langle c_{k}^{\dagger} c_{k}\right\rangle$, $C_{\rho}\left(k-k^{\prime}\right)$, etc., which appear in Eqs. (21) - (23), to find new values for the renormalized coupling parameters. This procedure is repeated until the self-consistency is reached. Note that the Hamiltonian $\tilde{\mathcal{H}}_{f}$ for the localized electrons is usually denoted as the lattice gas model. Due to the dependence of $\tilde{g}_{i j}$ on $i-j$, the interaction in $\tilde{\mathcal{H}}_{f}$ is not necessarily restricted to nearest neighbors.

Let us add two side remarks: The first one concerns the validity of the present approach. As is obvious from the renormalization procedure in Sect. II we are here dealing with a weak-coupling approach. The Coulomb interaction $U$ is taken as the 'small' quantity which is successively integrated out in the procedure. However in each renormalization step not only the lowest order in $U$ is included. Instead, certain renormalization contributions up to infinite order in $U$ are taken into account. Therefore, we expect the present approach to be valid for $U$ values which are less or at most of the order of the hopping term $t$. For larger values of $U$ either newly generated renormalization terms would have to be added. Or, more appropriate for large $U$ would be to take the dominant $U$ term as part of a new 'unperturbed' Hamiltonian $\mathcal{H}_{0}$ and the hopping term as part of the new perturbation $\mathcal{H}_{1}$.

The second remark applies to the optimal choice of the renormalization interval $\Delta \lambda$. It is best determined by the size of the system, i.e., by the requirement that the 'energy shell' contains only a few $k$-points. Thus, the number of $\Delta \lambda$ intervals should be of the order of the lattice size $N$. For a larger number of intervals the result would be the same since more steps occur without renormalization since no transition energies fit into the interval. On the 
other hand a smaller number of $\Delta \lambda$ intervals leads to a quite large renormalization in some steps since many $k$-points could contribute in these intervals. In practise, for a given $N$ we have increased the number of intervals until the renormalization does not change any more.

\section{EVALUATION OF STATIC EXPECTATION VALUES}

The renormalization equations depend on expectation values $\left\langle n_{i}\right\rangle,\left\langle n_{i} n_{j}\right\rangle$ and $\left\langle c_{k}^{\dagger} c_{k}\right\rangle$ which have been defined with the full Hamiltonian $\mathcal{H}$. Since thermal averages are invariant with respect to a unitary transformations, we can do the averaging with the renormalized Hamiltonian $\tilde{\mathcal{H}}$, provided we also transform the operators,

$$
\langle\mathcal{A}\rangle=\langle\mathcal{A}(\lambda)\rangle_{\mathcal{H}_{\lambda}}=\langle\mathcal{A}(\lambda \rightarrow 0)\rangle_{\tilde{\mathcal{H}}}, \quad \mathcal{A}(\lambda)=e^{X_{\lambda}} \mathcal{A} e^{-X_{\lambda}}
$$

To evaluate $\left\langle c_{k}^{\dagger} c_{k}\right\rangle$ one needs the renormalization equations for the operators $\left(c_{k}^{\dagger} c_{k}\right)(\lambda)=$ $c_{k}^{\dagger}(\lambda) c_{k}(\lambda)$, where $c_{k}(\lambda)=e^{X_{\lambda, \Delta \lambda}} c_{k} e^{-X_{\lambda, \Delta \lambda}}$. For $c_{k}(\lambda)$ we use the following ansatz,

$$
c_{k}(\lambda)=\alpha_{k, \lambda} c_{k}+\frac{1}{N} \sum_{k^{\prime}, i} \beta_{k, k^{\prime}, \lambda} e^{i\left(k-k^{\prime}\right) R_{i}} \delta n_{i} c_{k^{\prime}},
$$

where $\alpha_{k, \lambda}$ and $\beta_{k, k^{\prime}, \lambda}$ are $\lambda$-dependent coefficients. The operator structure of $c_{k}(\lambda)$ in (30) is again taken from the first order expansion in $U$. All higher order terms are neglected. The renormalization equations for the new parameters can be derived in close analogy to the renormalization of $\mathcal{H}_{\lambda}$. One finds,

$$
\begin{aligned}
\alpha_{k,(\lambda-\Delta \lambda)} & =\alpha_{k, \lambda}-\alpha_{k, \lambda} \sum_{k^{\prime}}\left[1-\cos \left(A_{k k^{\prime}}^{\lambda, \Delta \lambda} \sqrt{\frac{C_{\rho}\left(k-k^{\prime}\right)}{N}}\right)\right] \Theta_{k k^{\prime}}^{\lambda, \Delta \lambda} \\
& +\frac{1}{\sqrt{N}} \sum_{k^{\prime}} \beta_{k k^{\prime}, \lambda} \Theta_{k k^{\prime}}^{\lambda, \Delta \lambda} \sqrt{C_{\rho}\left(k-k^{\prime}\right)} \sin \left(A_{k k^{\prime}}^{\lambda, \Delta \lambda} \sqrt{\frac{C_{\rho}\left(k-k^{\prime}\right)}{N}}\right)
\end{aligned}
$$

and

$$
\begin{aligned}
\beta_{k k^{\prime},(\lambda-\Delta \lambda)} & \left.=\beta_{k k^{\prime}, \lambda}-\alpha_{k, \lambda} \sqrt{\frac{N}{C_{\rho}\left(k-k^{\prime}\right)}} \sin \left(A_{k k^{\prime}}^{\lambda, \Delta \lambda} \sqrt{\frac{C_{\rho}\left(k-k^{\prime}\right)}{N}}\right)\right] \Theta_{k k^{\prime}}^{\lambda, \Delta \lambda} \\
& -\beta_{k k^{\prime}, \lambda}\left(1-\cos \left[A_{k k^{\prime}}^{\lambda, \Delta \lambda} \sqrt{\frac{C_{\rho}\left(k-k^{\prime}\right)}{N}}\right]\right) \Theta_{k k^{\prime}}^{\lambda, \Delta \lambda}
\end{aligned}
$$

where $A_{k, k^{\prime}}^{\lambda, \Delta \lambda}$ and $C_{\rho}\left(k-k^{\prime}\right)$ are defined by Eq. (25) and (24), respectively. The initial values are again those of the original model $(\lambda=\Lambda), \alpha_{k, \Lambda}=1, \beta_{k k^{\prime}, \Lambda}=0$. Eqs. (30) -(32) determine 
the transformation behavior of $c_{k}(\lambda)$. By the use of (30) we finally obtain

$$
n_{k}=\left\langle c_{k}^{\dagger} c_{k}\right\rangle=\left|\tilde{\alpha}_{k}\right|^{2}\left\langle c_{k}^{\dagger} c_{k}\right\rangle_{\tilde{\mathcal{H}}_{c}}+\frac{1}{N} \sum_{k^{\prime}}\left|\tilde{\beta}_{k, k^{\prime}}\right|^{2} C_{\rho}\left(k-k^{\prime}\right)\left\langle c_{k^{\prime}}^{\dagger} c_{k^{\prime}}\right\rangle_{\tilde{\mathcal{H}}_{c}}
$$

where $\tilde{\alpha}_{k}=\alpha_{k,(\lambda=0)}$ and $\tilde{\beta}_{k, k^{\prime}}=\beta_{k k^{\prime},(\lambda=0)}$, and $\left\langle c_{k}^{\dagger} c_{k}\right\rangle_{\tilde{\mathcal{H}}_{c}}$ is the Fermi function formed with $\tilde{\mathcal{H}}_{c},\left\langle c_{k}^{\dagger} c_{k}\right\rangle_{\tilde{\mathcal{H}}_{c}}=\left(e^{\beta \tilde{\varepsilon}_{k}}+1\right)^{-1}$.

The occupation number operators $n_{i}$ of the localized electrons commute with $X_{\lambda}$. Thus $n_{i}$ will not be renormalized, $n_{i}(\lambda)=n_{i},\left(n_{i} n_{j}\right)(\lambda)=n_{i} n_{j}$, i.e.,

$$
n_{f}=\left\langle n_{i}\right\rangle=\left\langle n_{i}\right\rangle_{\tilde{\mathcal{H}}_{f}} \quad\left\langle\delta n_{i} \delta n_{j}\right\rangle=\left\langle\delta n_{i} \delta n_{j}\right\rangle_{\tilde{\mathcal{H}}_{f}} \cdot
$$

Here the expectation values are formed with the lattice gas model $\tilde{\mathcal{H}}_{f}$ since the conduction and the localized part of the renormalized Hamiltonian $\tilde{\mathcal{H}}$ are decoupled. The expectation values in (34) can be found by direct numerical evaluation in the local occupation number representation.

\section{DYNAMIC CORRELATION FUNCTIONS}

Dynamical quantities can be evaluated by the PRM approach along the same lines. In what follows we shall discuss the spectral functions of the conduction and localized electrons. The one-particle spectral functions of conduction electrons $A_{k}^{+}(\omega)$ and $A_{k}^{-}(\omega)$ are defined as

$$
A_{k}^{+}(\omega)=\frac{1}{2 \pi} \int_{-\infty}^{\infty}\left\langle c_{k}(t) c_{k}^{\dagger}\right\rangle e^{i \omega t} d t, \quad A_{k}^{-}(\omega)=\frac{1}{2 \pi} \int_{-\infty}^{\infty}\left\langle c_{k}^{\dagger} c_{k}(t)\right\rangle e^{i \omega t} d t
$$

where $A_{k}^{+}(\omega)$ describes the creation of an electron $k$ at time zero and its annihilation at time $t$ whereas in $A_{k}^{-}(\omega)$ first an electron is annihilated. It is well-known that $A_{k}^{+}(\omega)$ and $A_{k}^{-}(\omega)$ can be measured by inverse photoemission (IPE) and by photoemission (PE) experiments. To evaluate $A_{k}^{+}(\omega)$ and $A_{k}^{-}(\omega)$ we again exploit the property (29) of expectation values and the ansatz (30) for $c_{k}(\lambda)$ and $c_{k}^{\dagger}(\lambda)$. We find

$$
\begin{aligned}
& A_{k}^{+}(\omega)=\left|\tilde{\alpha}_{k}\right|^{2}\left(1-\left\langle c_{k}^{\dagger} c_{k}\right\rangle_{\tilde{\mathcal{H}}_{c}}\right) \delta\left(\omega-\tilde{\varepsilon}_{k}\right)+\frac{1}{N} \sum_{k^{\prime}}\left|\tilde{\beta}_{k k^{\prime}}\right|^{2} C_{\rho}\left(k-k^{\prime}\right)\left(1-\left\langle c_{k^{\prime}}^{\dagger} c_{k^{\prime}}\right\rangle_{\tilde{\mathcal{H}}_{c}}\right) \delta\left(\omega-\tilde{\varepsilon}_{k^{\prime}}\right) \\
& A_{k}^{-}(\omega)=\left|\tilde{\alpha}_{k}\right|^{2}\left\langle c_{k}^{\dagger} c_{k}\right\rangle_{\tilde{\mathcal{H}}_{c}} \delta\left(\omega-\tilde{\varepsilon}_{k}\right)+\frac{1}{N} \sum_{k^{\prime}}\left|\tilde{\beta}_{k k^{\prime}}\right|^{2} C_{\rho}\left(k-k^{\prime}\right)\left\langle c_{k^{\prime}}^{\dagger} c_{k^{\prime}}\right\rangle_{\tilde{\mathcal{H}}_{c}} \delta\left(\omega-\tilde{\varepsilon}_{k^{\prime}}\right)
\end{aligned}
$$


where $\left\langle c_{k^{\prime}}^{\dagger} c_{k^{\prime}}\right\rangle_{\tilde{\mathcal{H}}_{c}}$ is the Fermi distribution. Note that in (36) we have already reduced the cutoff to $\lambda \rightarrow 0$. The first term in $A_{k}^{+}(\omega)$ and $A_{k}^{-}(\omega)$ describes the coherent oneelectron excitation which is the only excitation in a free electron gas model. The remaining contributions are incoherent excitations due to the coupling of $c_{k}$ to different conduction electrons with wave vectors $k^{\prime}$. The coupling is mediated by density fluctuations of local electrons which lead to the appearance of the charge susceptibility $C_{\rho}\left(k-k^{\prime}\right)$ of the localized electrons in (36).

The spectral sum rule can be found by integrating $\left(A_{k}^{+}(\omega)+A_{k}^{-}(\omega)\right)$ over $\omega$ and using $\left[c_{k}^{\dagger}, c_{k}\right]_{+}=1$

$$
1=\int_{-\infty}^{\infty} d \omega\left(A_{k}^{+}(\omega)+A_{k}^{-}(\omega)\right)=\left|\tilde{\alpha}_{k}\right|^{2}+\frac{1}{N} \sum_{k^{\prime}}\left|\tilde{\beta}_{k k^{\prime}}\right|^{2} C_{\rho}\left(k-k^{\prime}\right) .
$$

This relation can also be derived directly from (30).

Next, we evaluate the averaged one-particle spectral functions $B^{+}(\omega)$ and $B^{-}(\omega)$ of localized electrons,

$$
B^{+}(\omega)=\frac{1}{2 \pi} \int_{-\infty}^{\infty}\left\langle f_{i}(t) f_{i}^{\dagger}\right\rangle e^{i \omega t} d t \quad B^{-}(\omega)=\frac{1}{2 \pi} \int_{-\infty}^{\infty}\left\langle f_{i}^{\dagger} f_{i}(t)\right\rangle e^{i \omega t} d t
$$

Since the local $f$-charge is a constant of motion, all correlation functions between different sites $i$ and $j$ vanish. For the evaluation we again use (29) and the following ansatz for the transformed $f$-operator

$$
f_{i}(\lambda)=\nu_{\lambda} f_{i}+\frac{1}{N} \sum_{k k^{\prime}} \mu_{k, k^{\prime}, \lambda} e^{i\left(k-k^{\prime}\right) R_{i}} \delta\left(c_{k}^{\dagger} c_{k^{\prime}}\right) f_{i}
$$

where $\nu_{\lambda}$ and $\mu_{k, k^{\prime}, \lambda}$ are the $\lambda$-dependent coefficients. As before, the operator structure of the second term is assumed to agree with the one obtained from the first order contribution in $U$ and higher order terms have been neglected. The initial parameter values are $(\lambda=\Lambda)$ : $\nu_{\Lambda}=1, \mu_{k k^{\prime}, \Lambda}=0$. The renormalization equations for $\nu_{\lambda}$ and $\mu_{k, k^{\prime}, \lambda}$ can easily be derived. In the limit $\lambda \rightarrow 0$ we find the following result

$$
\begin{aligned}
& B^{+}(\omega)=\tilde{\nu}^{2} B_{0}^{+}(\omega)+\frac{1}{N^{2}} \sum_{k k^{\prime}}\left|\tilde{\mu}_{k k^{\prime}}\right|^{2} f\left(\tilde{\varepsilon}_{k}\right)\left(1-f\left(\tilde{\varepsilon}_{k^{\prime}}\right)\right) B_{0}^{+}\left(\omega+\tilde{\varepsilon}_{k}-\tilde{\varepsilon}_{k^{\prime}}\right) \\
& B^{-}(\omega)=\tilde{\nu}^{2} B_{0}^{-}(\omega)+\frac{1}{N^{2}} \sum_{k k^{\prime}}\left|\tilde{\mu}_{k^{\prime} k}\right|^{2} f\left(\tilde{\varepsilon}_{k}\right)\left(1-f\left(\tilde{\varepsilon}_{k^{\prime}}\right)\right) B_{0}^{-}\left(\omega-\left(\tilde{\varepsilon}_{k}-\tilde{\varepsilon}_{k^{\prime}}\right)\right) .
\end{aligned}
$$


We have introduced new correlation functions

$$
B_{0}^{+}(\omega)=\frac{1}{2 \pi} \int_{-\infty}^{\infty} d t e^{i \omega t}\left\langle f_{i}(t) f_{i}^{\dagger}\right\rangle_{\tilde{\mathcal{H}}_{f}} \quad B_{0}^{-}(\omega)=\frac{1}{2 \pi} \int_{-\infty}^{\infty} d t e^{i \omega t}\left\langle f_{i}^{\dagger} f_{i}(t)\right\rangle_{\tilde{\mathcal{H}}_{f}} .
$$

which are defined with the lattice gas model $\tilde{\mathcal{H}}_{f}$. In Appendix B the functions $B_{0}^{ \pm}(\omega)$ are evaluated by use of the retarded one- $f$ electron Green function $\mathcal{G}_{0}(\omega)$, defined with $\tilde{\mathcal{H}}_{f}$,

$$
\mathcal{G}_{0}(\omega)=i \int_{0}^{\infty} d t e^{i(\omega+i \eta) t}\left\langle\left[f_{i}(t), f_{i}^{\dagger}\right]_{+}\right\rangle_{\tilde{\mathcal{H}}_{f}} \quad\left(\eta=0^{+}\right) .
$$

\section{RESULTS}

\section{A. Static properties}

We now discuss the results obtained by numerical evaluation of the renormalization equations for the one-dimensional lattice. Note that in the following all energy values are given in units of the hopping matrix element $t$. One first has to calculate the fully renormalized parameters $\tilde{\varepsilon}_{k}, \tilde{\varepsilon}_{f}$, and $\tilde{g}_{i j}$ which determine the effective Hamiltonian $\tilde{\mathcal{H}}=\tilde{\mathcal{H}}_{c}+\tilde{\mathcal{H}}_{f}+\tilde{E}$ of (28). All static expectation values which enter the renormalization equations are evaluated self-consistently with the full Hamiltonian, following the procedure outlined in Sections III and IV. The effective Hamiltonian $\tilde{\mathcal{H}}_{f}$ describes the lattice gas model of ions which interact via a possibly long range interaction $\tilde{g}_{i j}$. Thus, we use an additional Monte Carlo simulation to evaluate the expectation values $n^{f}=\left\langle n_{i}\right\rangle_{\tilde{\mathcal{H}}_{f}}$ and $C_{f f}(\kappa)=\left\langle\delta n_{i} \delta n_{j}\right\rangle=\left\langle\delta n_{i} \delta n_{j}\right\rangle_{\tilde{\mathcal{H}}_{f}}$, which enter the renormalization equations together with $\left\langle c_{k}^{\dagger} c_{k}\right\rangle_{\tilde{\mathcal{H}}_{c}}=\left[1+e^{\beta \tilde{\varepsilon}_{k}}\right]^{-1}$.

As already mentioned, we start by making a guess for the initial values of $n_{f}, C_{\rho}\left(k-k^{\prime}\right)$ and $\left\langle c_{k}^{\dagger} c_{k}\right\rangle$, and calculate the renormalized parameters for $\lambda \rightarrow 0$. That is, we find $\tilde{\varepsilon}_{f}=\varepsilon_{f, \lambda=0}, \tilde{\varepsilon}_{k}=\varepsilon_{k, \lambda=0}$, and $\tilde{g}_{q}=g_{q, \lambda=0}$ by solving numerically the renormalization equations (21)-(23). Next, using the effective coupling $\tilde{g}_{q}$ we find the new values of the spatial interactions $\tilde{g}_{i j}$ and then recalculate the localized electron expectation values $n_{f}$ and $C_{f f}(\kappa)$ using a classical Monte Carlo simulation. The Fourier transform of $C_{f f}(\kappa)$ gives an approximate expression for $C_{\rho}\left(k-k^{\prime}\right)$. Using new values of $n_{f}, C_{\rho}\left(k-k^{\prime}\right)$ and $\left\langle c_{k}^{\dagger} c_{k}\right\rangle$ we iterate (21) - (23) until a self-consistent solution is reached.

In the following we consider only the one-dimensional Falicov-Kimball model at half- 
filling,

$$
n_{f}+n_{c}=1
$$

where $n_{f}=\left\langle n_{i}\right\rangle$ and $n_{c}=1 / N \sum_{k}\left\langle c_{k}^{\dagger} c_{k}\right\rangle$ is the averaged local occupation numbers of conduction electrons. Condition (44) represents a point of special interest for valence and possibly metal-insulator transitions, caused by the promotion of electrons from localized $f$ orbitals $\left(f^{n} \rightarrow f^{n+1}\right)$ to the conduction band states ${ }^{15}$.

\section{Temperature dependence}

In Fig. 1 the averaged local $f$-occupation number $n_{f}$ and the renormalized $f$-energy $\tilde{\varepsilon}_{f}$ from the PRM approach are shown as function of temperature for $U=1.0$ and several values of unrenormalized $f$-level position $\varepsilon_{f}$. Note, the chemical potential $\mu$ has to be adjusted at each temperature $T$, so as to fulfill condition (44). In the evaluation procedure this requires extra numerical cycles, in addition to those from the self-consistent determination of the expectation values. Therefore, only a rather small system of 20 lattice sites has been used to generate Fig. 1. Also the considerably increased effort in the MC sampling for the lattice gas part in $\mathcal{H}_{0, \lambda}$ at higher temperatures suggest to restrict oneself to a rather small system. This will be discussed below in some more detail. As expected, for small values of the bare $f$-level $\varepsilon_{f}$ (dashed curve in Fig. 1a), the average $f$-occupation $n_{f}$ increases with decreasing temperature and tends to 1 at $T=0$. The corresponding values of the renormalized $f$-level remain below the chemical potential $\left(\tilde{\varepsilon}_{f}<0\right.$ in Fig. 1b), so that all the sites are occupied with one $f$-electron. In contrast, for large values of $\varepsilon_{f}$ (solid curves) $n_{f}$ tends to zero for $T \rightarrow 0$ and $\tilde{\varepsilon}_{f}$ stays above the chemical potential $\left(\tilde{\varepsilon}_{f}>0\right)$. Thus all the $f$-sites are empty and the renormalized conduction electron band is completely filled. Finally, at intermediate values of the bare $\varepsilon_{f}$-value (dashed dotted curves) the $f$ occupation at $T=0$ tends to some value between 0 and 1 , signalizing an intermediate $f$-valence. In that case, the renormalized $f$-level approaches the chemical potential $\left(\tilde{\varepsilon}_{f}=0\right)$ at $T=0$. Below the chemical potential all the conduction electron states are occupied and $f$-states only partially, due to the condition $n_{f}+n_{c}=1$. 

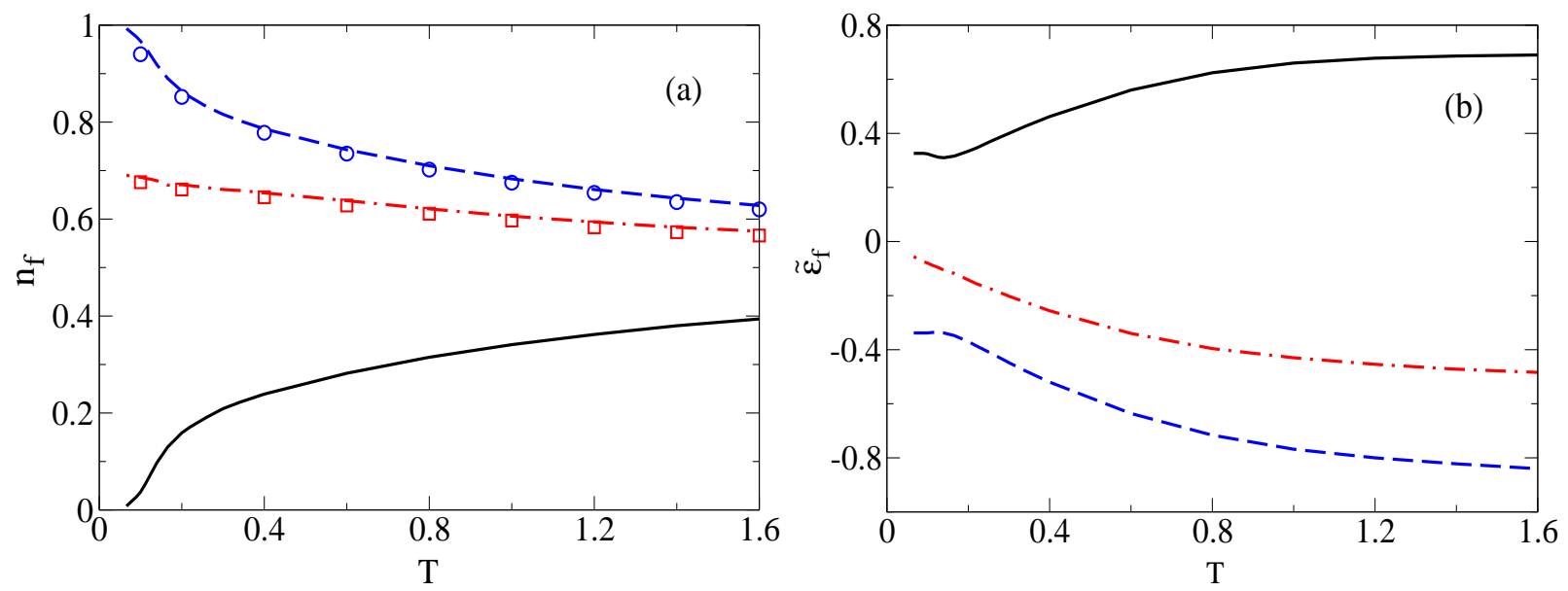

FIG. 1: (a) $f$-electron occupation number $n_{f}$ as function of temperature for $U=1.0$ and for three different values of the bare $f$-level $\varepsilon_{f}=0.5$ (dashed), $\varepsilon_{f}=1.2$ (dashed dotted), and $\varepsilon_{f}=3.5$ (solid). The open circles $\left(\varepsilon_{f}=0.5\right)$ and open squares $\left(\varepsilon_{f}=1.2\right)$ refer to ED results of reference $\underline{16}$. Note the good agreement with the PRM results. The values of $\varepsilon_{f}$ are measured from the lower edge of the conduction band. (b) Renormalized $f$-electron energy $\tilde{\varepsilon}_{f}$ as function of temperature for the same values of $U$ and $\varepsilon_{f}$-values as in panel (a).

\section{Low temperature properties at fillings $n_{f}=1 / 2$ and $n_{f}=1 / 3$}

Next, we discuss static properties for $n_{f}=1 / 2$ and $1 / 3$ at low temperatures. The parameters $\mu$ and $\varepsilon_{f}$ have to be adjusted in such a way that the condition (44) is fulfilled for a given value of $n_{f}$. Note that in a Monte Carlo simulation a nonzero temperature has to be used, which is set equal to $k_{B} T=0.1$. Also the most dominant ground-state configurations have been incorporated in the Monte-Carlo simulation so that the system size could be extended to $N=150$ sites. For $n_{f}=1 / 2$ the chemical potential $\mu$ is in the middle of the renormalized conduction band $\tilde{\varepsilon}_{k}$.

In Fig. 2r the result for $n_{k}$ obtained from the PRM is plotted as function of $k$ for $n_{c}=1 / 2$ and for different values of the Coulomb repulsion $U$. The results for $n_{k}$ from the PRM approach are very similar to those from Ref. $\frac{17}{}$ (for the same values of $U$ ).

In Fig. 2a the renormalized one-particle energy $\tilde{\varepsilon}_{k}$ is shown as function of momentum $k$ for $n_{c}=1 / 2\left(n_{f}=1 / 2\right)$, and for the same interaction values $U$ as in Fig. 2r. Note, that $\tilde{\varepsilon}_{k}$ can be considered as the excitation energy of a quasiparticle of the original interacting model 

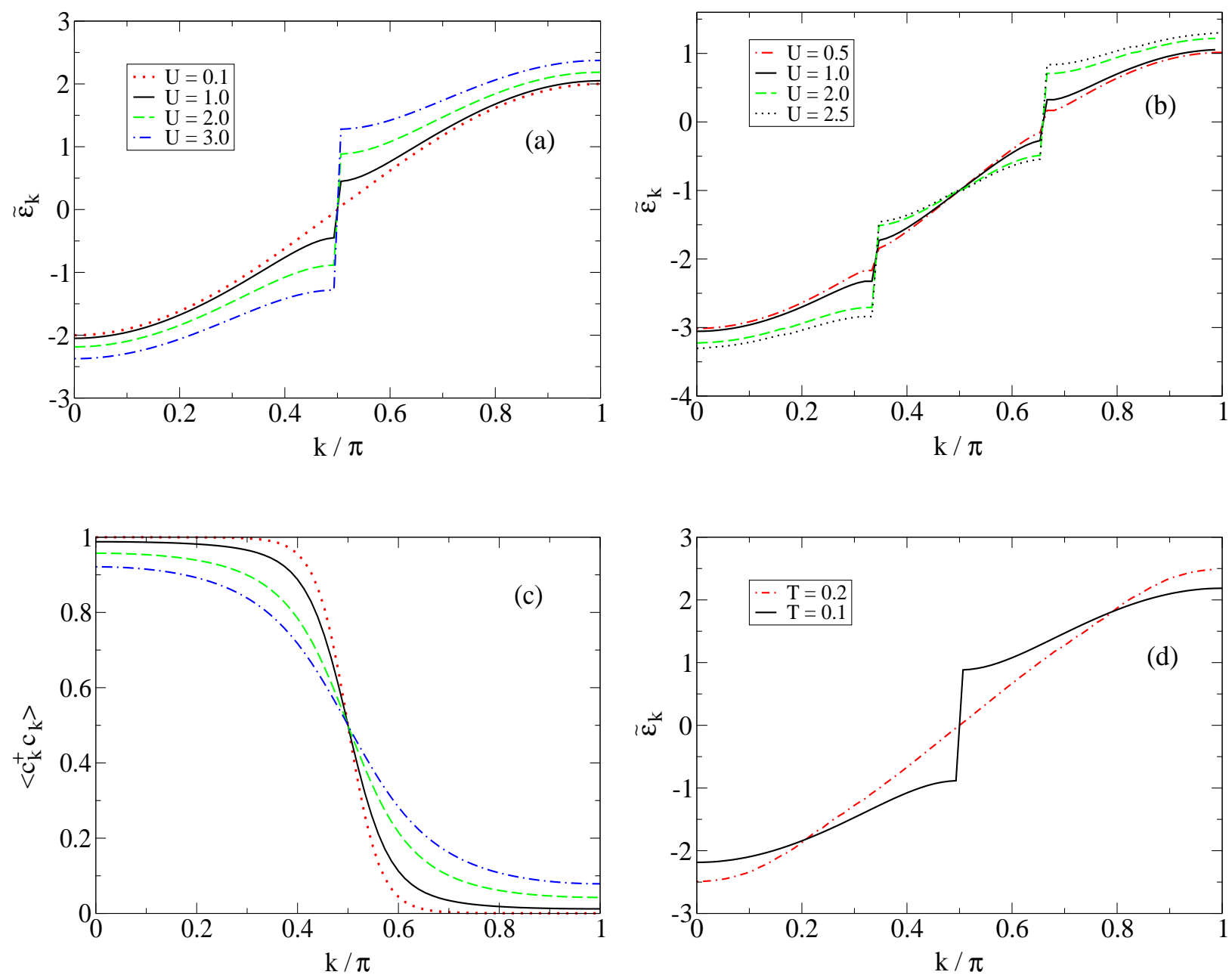

FIG. 2: (a) Renormalized conduction electron energy $\tilde{\varepsilon}_{k}$ for different Coulomb repulsions $U$ (= $0.1,1.0,2.0,3.0)$ and $n_{c}=n_{f}=1 / 2$. Note the appearance of a gap of magnitude $U$ at the Fermi momentum $k_{F}=\pi / 2$. (b) Same quantity $\tilde{\varepsilon}_{k}$ for $n_{c}=2 / 3\left(n_{f}=1 / 3\right)$ for $U=0.5,1.0,2.0,2.5$. $\tilde{\varepsilon}_{k}$ shows two gaps where the one at the Fermi momentum $k_{F}=(2 / 3) \pi$ is now smaller than $U$.(c) Momentum distribution $n_{k}=\left\langle c_{k}^{\dagger} c_{k}\right\rangle$ for the same parameters as in panel (a) and $n_{f}=n_{c}=1 / 2$. The smooth behavior of $n_{k}$ at the Fermi momentum follows from the gap in $\tilde{\varepsilon}_{k}$. (d) $\tilde{\varepsilon}_{k}$ for two different temperatures $T=0.1$ (solid line) and $T=0.2$ (dashed dotted line) and $U=2.0, n_{f}=1 / 2$. Note that the gap at $k_{F}$ for $T=0.1$ is completely smeared out for the higher temperature $T=0.2$.

(11). This follows from the diagonal form of $\tilde{\mathcal{H}}_{c}$ in $(28)$ and the fact that $\tilde{\mathcal{H}}$ is obtained from the original model by a unitary transformation. The main feature of $\tilde{\varepsilon}_{k}$ is the appearance of a gap at the Fermi level, which opens for any, even small, value of the repulsion energy $U$. Such a gap was recently anticipated by Farkasovsky ${ }^{17}$ from the correspondence of the 
Falicov-Kimball model to the Tomonaga-Luttinger fermions. There, the smooth behavior of $n_{k}$ with $k$ also gives rise to a gap in the charge excitations. Note that for $n_{c}=2 / 3$ $\left(n_{f}=1 / 3\right)$ (Fig. 2b) again a gap opens at the Fermi energy for $k_{F}=(2 / 3) \pi$ for all values of $U$. Thus, the Luttinger theorem is fulfilled even for the present case of an insulating state. By closer inspection of Figs. $2 \mathrm{a}, \mathrm{b}$ one finds that the gaps at $k_{F}$ scale linearly with $U$ both for $n_{c}=1 / 2$ and $n_{c}=2 / 3$. The proportionality constant is almost 1 for $n_{c}=1 / 2$ whereas for $n_{c}=2 / 3$ it is somewhat smaller than 1 .
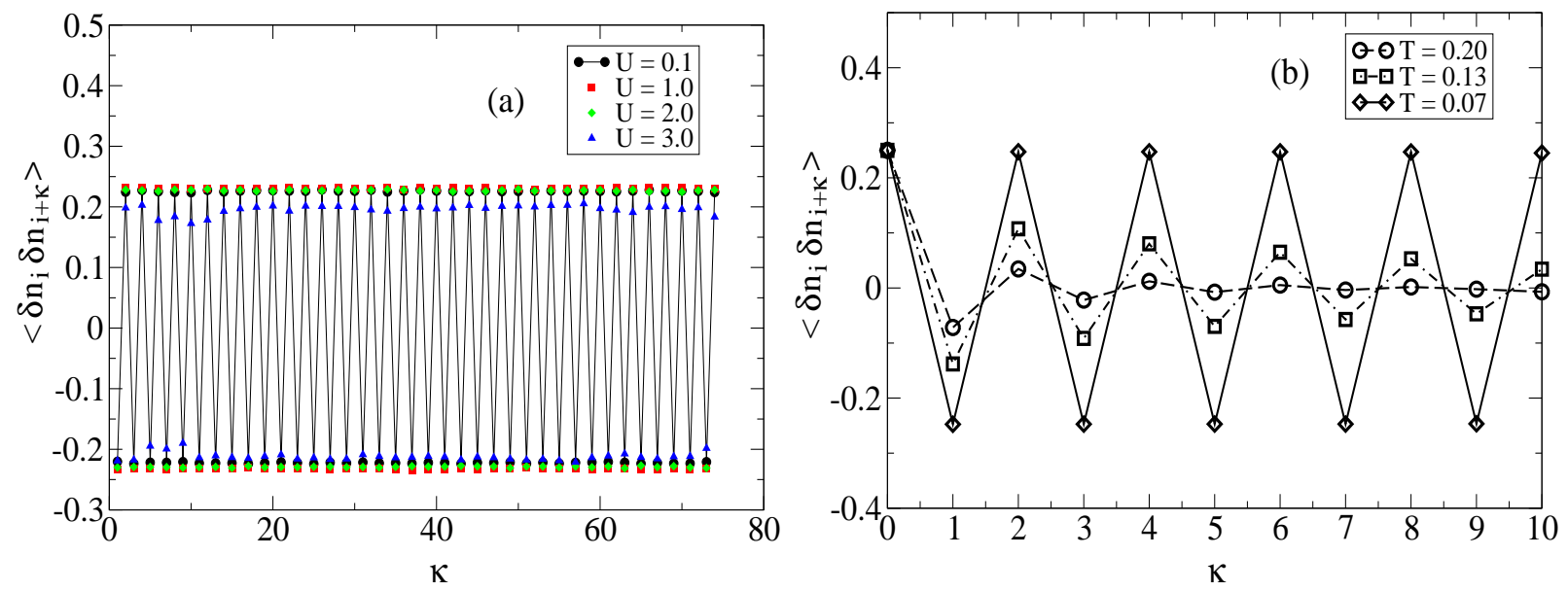

FIG. 3: (a) Correlation function $C_{f f}(\kappa)=\left\langle\delta n_{i} \delta n_{i+\kappa}\right\rangle$ of localized electrons for $n_{f}=1 / 2$. For $n_{f}=1 / 3$ we obtain an analogous result. The length $\kappa$ characterizes the distance between the sites. The results correspond to averages $(1 / N) \sum_{i}\left\langle\delta n_{i} \delta n_{i+\kappa}\right\rangle$ over all lattice sites (see text). (b) Correlation function $C_{f f}(\kappa)=\left\langle\delta n_{i} \delta n_{i+\kappa}\right\rangle$ of localized electrons for a chain with 20 lattice sites for three different temperatures $T=0.20,0.13,0.07$ and $U=1.0$ and $n_{f}=1 / 2$.

Fig. 3h shows the spatial correlation functions $C_{f f}(\kappa)=\left\langle\delta n_{i} \delta n_{i+\kappa}\right\rangle$ between the localized electrons for the same parameters as in Fig. 2a. The index $\kappa$ denotes the distance between the two involved lattice sites. Note that for $n_{f}=1 / 2$ the values of the correlation function are always the same at every second site $(\kappa=2,4,6, \cdots)$ whereas for all sites in between $(\kappa=1,3,5, \cdots)$ they differ only by a minus sign. 


\section{Discussion}

Note that the long-range correlations develop already for arbitrarily small values of $U$. This behavior should be related to the long range order of the one-dimensional FalicovKimball model at zero temperature ${ }^{6}$. Note that in the present calculations the temperature is chosen small but finite $\left(k_{B} T=0.1\right)$, so that in the strict sense no infinitely long range order exists. The origin of the long-range correlations can easily be understood from a low order $U$ expansion of the effective $f$-coupling. For the Fourier transform $g_{q}$ of the local quantity $g_{i j}$ one finds by use of (23), (24) and (25)

$$
\begin{aligned}
g_{q,(\lambda-\Delta \lambda)}=g_{q, \lambda}- & \frac{U^{2}}{2 N} \sum_{k} \frac{\left\langle c_{k}^{\dagger} c_{k}\right\rangle-\left\langle c_{k+q}^{\dagger} c_{k+q}\right\rangle}{\varepsilon_{k+q, \lambda}-\varepsilon_{k, \lambda}} \Theta_{k, k+q}^{\lambda, \Delta \lambda} \\
& +\frac{U^{2}}{2 N^{2}} \sum_{k, q^{\prime}} \frac{\left\langle c_{k}^{\dagger} c_{k}\right\rangle-\left\langle c_{k+q^{\prime}}^{\dagger} c_{k+q^{\prime}}\right\rangle}{\varepsilon_{k+q^{\prime}, \lambda}-\varepsilon_{k, \lambda}} \Theta_{k, k+q^{\prime}}^{\lambda, \Delta \lambda}
\end{aligned}
$$

where the last term excludes equal sites $i=j$ in $g_{i j}$. As follows from (45), the interaction between the localized electrons is caused from the coupling to particle-hole excitations $\varepsilon_{k+q, \lambda}-\varepsilon_{k, \lambda}$ where the wavevector runs over the whole Brillouin zone. Here, the energies $\varepsilon_{k, \lambda}$ and $\varepsilon_{k+q, \lambda}$ have to be either below or above the Fermi level, i.e. $|k|<k_{F}$ and $|k+q|>k_{F}$ or $|k|>k_{F}$ and $|k+q|<k_{F}$. Note that for $q=\pi$ and $n_{f}=1 / 2$ this condition is fulfilled for all $k$-values of the Brillouin zone so that the most dominant renormalization of $g_{q}$ occurs for $q=\pi$. In contrast, for $q$-values different from $\pi$ always less $k$ terms contribute. For instance,

for $q \approx 0$ only $k$ points from the sum in (45) can contribute which are located in a small region around the Fermi momentum $k_{F}$. As a result of the dominant coupling $g_{q}$ at $q=\pi$ one is immediately lead to a infinitely ranged spatial correlation between the $f$-electrons having an alternating sign from site to site. This explains the long-range behavior in Fig. 3a. Note again that this feature is not based on a strong-coupling argument. Instead, the long-ranged correlations are observed already for small values for $U$.

The alternating behavior of $C_{f f}(\kappa)=\left\langle\delta n_{i} \delta n_{i+\kappa}\right\rangle$ with $\kappa$ can be interpreted as follows: At zero temperatures the model has two equivalent degenerate ground states of period two (for $n_{f}=1 / 2$ ), separated by a potential barrier, with the arrays $\{1010 \ldots 10\}$ and $\{0101 \ldots 01\}$ of the localized ions. Therefore, we might use the following ansatz for the state vector of 
the system at very low but finite temperatures,

$$
\left|\Phi_{g}^{(2)}\right\rangle=\frac{1}{\sqrt{2}}(|10101 \ldots 10\rangle+|0101 \ldots 01\rangle)
$$

In order to obey translation invariance the sum of the two generated ground states have been taken and ground-state fluctuations have been neglected. Note that the two states are never connected by nonvanishing matrix elements when physical quantities are evaluated. To proceed, we use (46) to evaluate the correlation function $\left\langle n_{i} n_{i+\kappa}\right\rangle$ of Fig. 3. As explained, the result corresponds to the average $(1 / N) \sum_{i}\left\langle n_{i} n_{i+\kappa}\right\rangle$ over all lattice sites and is independent of $i$. From ansatz (46) one finds

$$
\left\langle n_{i} n_{i+\kappa}\right\rangle=\left\{\begin{array}{l}
\frac{1}{2}: \kappa=2,4,6, \ldots \\
0: \kappa=1,3,5, \ldots
\end{array}\right.
$$

With $\left\langle n_{i}\right\rangle=1 / 2$ one obtains for $C_{f f}(\kappa)=\left\langle\delta n_{i} \delta n_{i+\kappa}\right\rangle$

$$
C_{f f}(\kappa)=\left\{\begin{aligned}
\frac{1}{4} & : \kappa=2,4,6, \ldots \\
-\frac{1}{4}: & \kappa=1,3,5, \ldots
\end{aligned}\right.
$$

Obviously the result (48) agrees very well with the outcome from the PRM approach according to Fig. 3a for small but also for rather large values of $U$. A similar reasoning may also be given for $n_{f}=1 / 3\left(n_{c}=2 / 3\right)$. Here, the degenerate ground-states have period three with arrays $\{100100 \ldots 100\},\{010010 \ldots 010\}$, and $\{001001 \ldots 001\}$ in analogy to (46).

Note that the amplitude of the $f$-correlation function $\left\langle\delta n_{i} \delta n_{i+\kappa}\right\rangle$ is almost independent of $U$. Already for small $U$-values the $f$-electrons are ordered according to the qualitative description of Fig. 3 a . The behavior of the conduction electrons is quite different. The correlation function $C_{c f}(\kappa)=\left\langle n_{i}^{c} n_{i+\kappa}\right\rangle$ describes the spatial correlation between a conduction electron at site $i$ and an $f$ electron at site $i+\kappa$. An evaluation for $C_{c f}(\kappa)$ correct up to first order in $U$ gives

$$
C_{c f}(\kappa)=\frac{n_{f}}{N} \sum_{k}\left|\tilde{\alpha}_{k}\right|^{2}\left\langle c_{k}^{\dagger} c_{k}\right\rangle_{\tilde{\mathcal{H}}_{c}}+\frac{2}{N}(-1)^{\kappa} \sum_{k} \tilde{\alpha}_{k} \tilde{\beta}_{k+Q}\left\langle c_{k}^{\dagger} c_{k}\right\rangle_{\tilde{\mathcal{H}}_{c}}+O\left(U^{2}\right)
$$

$(Q=\pi)$, where the renormalized parameter $\tilde{\beta}_{k+Q} \sim U$ is negative (compare (32)). Obviously, the second term in (49) describes an oscillating behavior with $\kappa$ of $c f$-correlations which favour the presence of conduction electrons in between the $f$ sites. However, in contrast to the oscillating behavior of $C_{f f}(\kappa)$, the amplitude of this charge-density wave-like 
behavior of the correlation function is proportional to $U$.

For an interpretation of the temperature dependence of $C_{f f}(\kappa)$ note that the two arrays $\{1010 \ldots 10\}$ and $\{0101 \ldots 01\}$ (for $n_{f}=1 / 2$ ) of the localized ions are equivalent to the two Néel states $|\uparrow \downarrow \uparrow \downarrow \cdots \uparrow \downarrow\rangle$ and $|\downarrow \uparrow \downarrow \uparrow \cdots \downarrow \uparrow\rangle$ of the one-dimensional Ising antiferromagnet. For the latter model the spatial spin correlation function $\left\langle S_{i}^{z} S_{i+\kappa}^{z}\right\rangle$ decays exponentially at low temperatures as $\exp (-\kappa / \xi)$ due to excitation of kinks or domain walls. The correlation length is given by $\xi \sim \exp \left(\beta\left|J_{z}\right| / 2\right)$, which shows that it becomes infinite only exactly at zero temperature. $J_{z} / 2$ is the characteristic energy for the creation of domain walls $\frac{18}{}$. For the one-dimensional Falicov-Kimball model a quite similar behavior is expected for the correlation function $C_{f f}(\kappa)$. Here, also domain wall states can be thermally excited. They are obtained by interchanging the positions of neighboring $f$ - and conduction electrons. In order to investigate the temperature dependence of the correlation length we have evaluated $C_{f f}(\kappa)=\left\langle\delta n_{i} \delta n_{i+\kappa}\right\rangle$ for different temperatures $T$ (Fig. 3 $\mathrm{b}$ ). Note that for higher temperatures excited states would have to be included in the MC-sampling which leads to considerable numerical increase. Therefore, instead of taking a system with 150 lattice sites as before a rather small system with only 20 sites was investigated where an exact diagonalization was performed. Fig. 3b shows that for the two higher temperatures $T=0.20$ and $T=0.13$ the correlation function decays roughly exponentially to zero within a few lattice sites whereas for the lowest temperature $T=0.07$ no decay is observed. Thus, in the latter case the correlation length $\xi$ obviously exceeds the length of the lattice. The same feature was already found in Fig. 3a, where no decay was observed up to $\kappa_{\max } \approx 70$. We can conclude that for the lowest temperature no excited states contribute to the correlation function for the given lattice sizes. Note that this result is not a consequence of approximations used in the present PRM approach. Instead at the lowest temperature the correlation length is larger than the lattice extension but should be still finite. Only exactly at $T=0$ the correlation length $\xi$ should be infinite. An equivalent behavior is also found for the itinerant electron-dispersion $\tilde{\varepsilon}_{k}$ as function of temperature. In Fig. $2 \mathrm{~d}$ the dispersion of $\tilde{\varepsilon}_{k}$ is shown for two temperatures $T=0.1$ and $T=0.2$ for $U=2.0$ and $n_{f}=1 / 2$. Whereas at $T=0.1$ the gap at $k_{F}$ is clearly seen it is interesting to note that it is already smeared out for $T=0.2$.

Note that an infinitely large correlation length $\xi$ leads for the Fourier transform $C_{\rho}(q)$ 
of $C_{f f}(\kappa)$ to $C_{\rho}(q) \sim N \delta_{q, \pi}$. Instead, for a finite correlation length $\xi$ one would find a 'softened' Kronneker function around $q=\pi$ within a range of the order of the inverse

correlation length $\xi^{-1}$. Thus the gap in the one-particle excitation energy $\tilde{\varepsilon}_{k}$ of Figs. 2a,b also softens over a range in $k$-space of the same order $\xi^{-1}$. It follows that the system is metallic at arbitrarily small temperature. The softening of the gap could hardly be seen in Figs. 2a,b for a correlation length larger than the lattice extension of, may be, 100 times the lattice constant. Thus, the results for $\tilde{\varepsilon}_{k}$ and other quantities are extremely good approximations also for the $T=0$ limit.

\section{B. Dynamical properties at low $T$}

Next, we evaluate the dynamical properties using the PRM approach. First we consider the electronic density of states (DOS) of conduction electrons

$$
\rho_{c}(\omega)=\frac{1}{N} \sum_{k}\left(A_{k}^{+}(\omega)+A_{k}^{-}(\omega)\right)=\frac{1}{N} \sum_{k} \frac{1}{2 \pi} \int_{-\infty}^{\infty}\left\langle\left[c_{k}(t), c_{k}^{\dagger}\right]_{+}\right\rangle e^{i \omega t} d t
$$

where $A_{k}^{+}(\omega)$ and $A_{k}^{-}(\omega)$ were defined in (35). The results from the PRM approach for the neutral case $n_{f}=n_{c}=1 / 2$ are shown in Figs. 4a and 4c for three different values of $U$. For small $U$ the DOS resembles that of free conduction electrons in one dimension with a divergence at the lower and upper band edge (dotted line of Fig. 4 a). For larger $U$ the opening of a charge gap of order $U$ around the Fermi level at $\omega=0$ can be observed (Fig. 4a and Fig. 4c). For still larger $U$ only charge excitations away from the Fermi level are left. This behavior can easily be understood from the former expressions (36) for the two spectral functions. Note that for rather small values of $U$ the first terms in both equations are the dominant ones since the prefactors $\left|\tilde{\beta}_{\mathbf{k k}^{\prime}}\right|^{2}$ of the second terms are of order $U^{2}$. From the gap in the renormalized quasiparticle energy $\varepsilon_{k}$ of Fig. 2a the occurrence of a gap in $\rho_{c}(\omega)$ immediately follows. Moreover, since the dispersion of $\varepsilon_{k}$ around the Fermi momentum flattens with increasing $U$ also the peaks in the DOS at the edges of the gap can be explained. The result for the larger $U$ value in Fig. 4k show a similar behavior as for the lower U's. Note however, for these cases our weak-coupling approach might be at the limit of its validity.

In Fig. 4a also the exact result for the DOS from Ref. ${ }^{21}$ at temperature $T=0$ is shown 

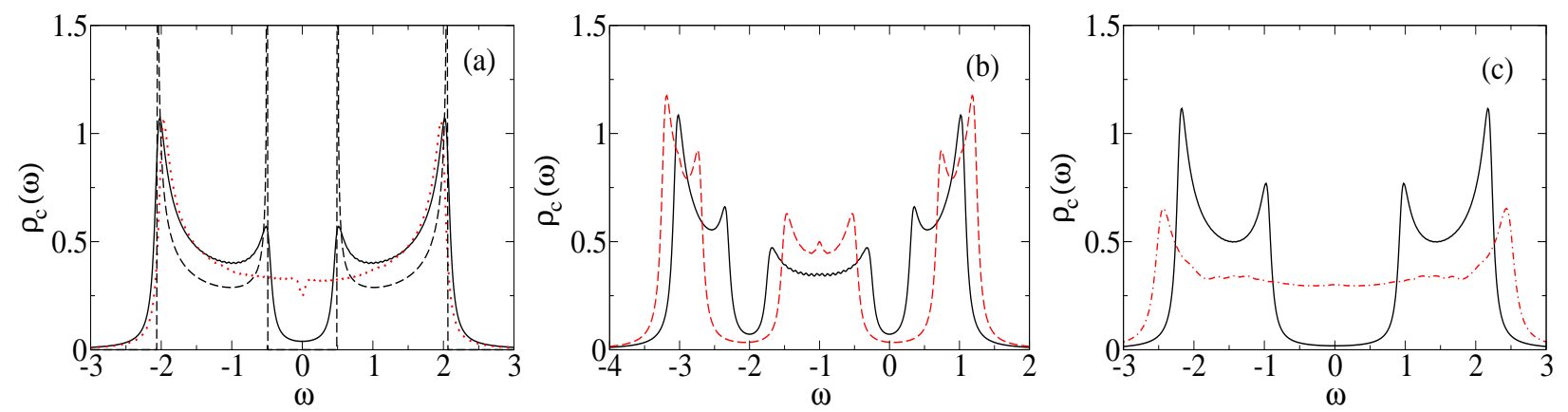

FIG. 4: (a) DOS $\rho_{c}(\omega)$ as function of $\omega$ for $U=0.1$ (dotted line) and $U=1.0$ (solid line) and $n_{f}=n_{c}=1 / 2$. Note the opening of a gap in the excitation spectrum at the Fermi level $\omega=0$. The dashed line is the exact result for $T=0$ as taken from Ref 21 . (b) DOS $\rho_{c}(\omega)$ for a different filling $n_{f}=1 / 3\left(n_{c}=2 / 3\right)$ for two values $U=1.0$ (solid line) and $U=2.0$ (dashed line). There are now two gaps found in the charge spectrum. (c) Same quantity as in panel (a) with $n_{f}=n_{c}=1 / 2$ for a larger $U$ value $U=2.0$ (solid line). Besides the low temperature result the DOS is also shown for a higher temperature $T=0.2$ (dashed dotted line). Note that the low-temperature gap at $\omega=0$ is smeared out for $T=0.2$.

(dashed line). There, the divergencies at the band edges appear since an infinitely large system size was considered in that approach. In contrast the renormalization equations in the PRM approach were solved on a finite lattice by which the divergencies become smooth. On the other hand, in order to satisfy the sum rule the spectral function within the bands is enlarged in the PRM result. In a recent Monte-Carlo study $\underline{19}$ the two-dimensional FalicovKimball model for the same concentrations $n_{f}=n_{c}=1 / 2$ was discussed. It shows quite similar features for the DOS as our PRM result for dimension $d=1$. In addition in Ref. $\underline{19}^{-1}$ also temperature-dependent effects have been discussed.

In Fig. $4 \mathrm{~b}$ the DOS $\rho_{c}(\omega)$ is shown for $n_{f}=1 / 3\left(n_{c}=2 / 3\right)$ for our lowest temperature $T=0.1$. As expected from the previous results for $\tilde{\varepsilon}_{k}$ in Fig. 2 $\mathrm{b}$, there are now two gaps found in the charge excitation spectrum, one of which is again located at the Fermi level. Note that in Fig. 4b the density of states is symmetric with respect to the band center. This symmetry can easily be understood as follows: First, at low temperatures the correlation function $\left\langle\delta n_{i} \delta n_{i+\kappa}\right\rangle$ is invariant with respect to translations by three lattice sites (compare the lower panel of Fig. 33). Therefore, $C_{\rho}\left(k-k^{\prime}\right) \sim N \delta_{k-k^{\prime}, \pm 2 \pi / 3}$ at low enough temperatures. By exploiting this relation and Eq. (22) one finds that all $k$ points which are symmetric to 
$\pm \pi / 2$ lead to the same renormalization of the quasiparticle energy $\varepsilon_{k, \lambda}$ but with an opposite sign. Thus, the renormalized quasiparticle energy $\tilde{\varepsilon}_{k}$ becomes antisymmetric, since also the original $\varepsilon_{k}$ was antisymmetric relative to $\pm \pi / 2$ and the DOS becomes symmetric relative to the band center in the present approach. This result is in contrast to the expectation that for occupation $n_{c}$ away from half-filling the DOS should be asymmetric. In fact, one can assure oneself that higher order terms to $X_{\lambda, \Delta \lambda}$ would give rise to a small additional asymmetric contribution to the DOS in our approach. Note that such a small asymmetry is found in an exact result $\underline{\underline{21}}$ for the DOS in one dimension at $T=0$ for $n_{c}=2 / 3$.

Finally, in Fig. 迎 also the influence of temperature on $\rho_{c}(\omega)$ is shown for $n_{f}=n_{c}=1 / 2$. Note that at $T=0.1$ the gap in the DOS is clearly seen. However, by increasing the temperature by a factor of 2 the gap becomes completely smeared out.
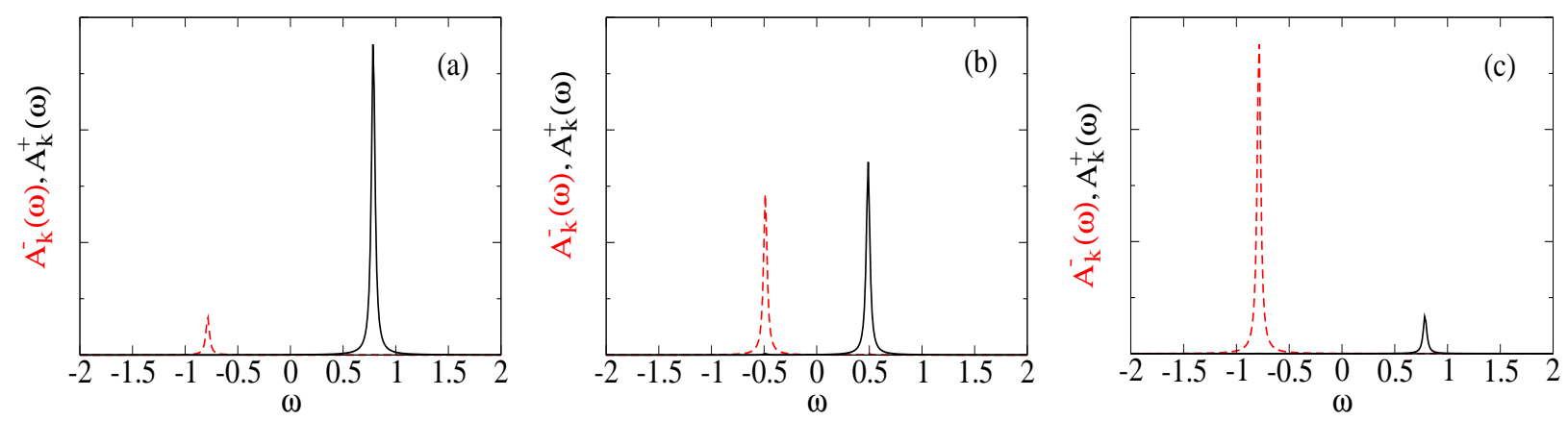

FIG. 5: One-particle spectral functions $A_{k}^{+}(\omega)$ (solid line) and $A_{k}^{-}(\omega)$ (dashed line) at low temperature $(T=0.1)$ as function of $\omega$ for different $k$ vectors, (a) $k>k_{F}$, (b) $k \geq k_{F}$, and (c) $k<k_{F}$. $\left(U=1.0, n_{f}=n_{c}=1 / 2.\right)$

In Figs. 5a-c the one-particle spectral functions $A_{k}^{+}(\omega)$ and $A_{k}^{-}(\omega)$ for different $k$ values are shown for the lowest possible temperature of $T=0.1$ and $n_{f}=n_{c}=1 / 2$. For $k>k_{F}$ (Fig. 5a) a coherent excitation is found in $A_{k}^{+}(\omega)$ and a small incoherent excitation in $A_{k}^{-}(\omega)$. To understand the latter feature, note that only a single $k^{\prime}$ value, $k^{\prime}=k-\pi$, contributes to the incoherent part in (36) due to $C_{\rho}\left(k-k^{\prime}\right) \sim N \delta_{k-k^{\prime}, \pi}$. The same remains true when $k$ comes closer to $k_{F}$ (Fig. [5b). In this case, however, the incoherent contribution from $A_{k}^{-}(\omega)$ has gained weight. The reason is the renormalization factor $\left|\tilde{\beta}_{k k^{\prime}}\right|^{2}$ in equation (36). It becomes stronger when $k$ and also $k^{\prime}$ approaches the Fermi momentum as follows from (32) and (25). Finally, for $k<k_{F}$ (Fig. 5: ) a coherent contribution is found in $A_{k}^{-}(\omega)$ and an 
incoherent excitation in $A_{k}^{+}(\omega)$.

In Figs. 6a-c the one-particle spectral functions $B^{+}(\omega)$ (black) and $B^{-}(\omega)$ (red) of the localized electrons averaged over all lattice sites from section IV are shown for different values of $U$ and $n_{f}=n_{c}=1 / 2$. For the two lower $U$-values $(U=0.1,1.0)$ at a rather low temperature $T=0.1$ the coherent parts (of weight $\tilde{\nu}^{2}$ ) dominate in Fig. 6 a the spectrum (compare (39)). In contrast, for the two larger $U$-values $(U=2.0,3.0)$ the incoherent excitations have gained considerable weight (Fig. 6b and Fig. 6c). Note that at a higher temperature $T=0.2$ the incoherent part is smeared out over a wide frequency range for $U=$ 2.0 (dashed dotted curve in Fig. 6b). The same feature would also be expected for $U=3.0$. However, no stable solution of the renormalization equations was found for this temperature. The discussed behavior can again be understood from the former PRM expressions (401), (41) for the spectral functions $B^{+}(\omega)$ and $B^{-}(\omega)$. The first term in both equations describes the coherent excitation of weight $\tilde{\nu}^{2}$ at $\omega \approx \tilde{\varepsilon}_{f}=0$ whereas the second term follows from the coupling of $f_{i}$ to electronic particle-hole excitations (compare (39)). Their weight $\tilde{\mu}_{k, k^{\prime}}^{2}$ is at least of order $U^{2}$. The gaps in Figs. 6a-c of order $U$ immediately follow from the gap in the quasiparticle energy $\tilde{\varepsilon}_{k}$.

The same quantities $B^{+}(\omega)$ (black) and $B^{-}(\omega)$ (red) are also shown in Fig. 7 for $f$-filling $n_{f}=1 / 3\left(n_{c}=2 / 3\right)$ and $U=1.0,2.0$. The gaps between the respective coherent and incoherent parts again correspond to the gap of the quasiparticle excitation in Fig. 20 at the Fermi level. Note that the spectrum is no longer symmetric around $\omega=0$. The different weights of $B^{+}(\omega)$ and $B^{-}(\omega)$ in Fig. 7 follow from the Fermi functions in (40) and (41).

We have also checked the $f$-sum rule

$$
\int_{\infty}^{\infty} d \omega\left(B^{+}(\omega)+B^{-}(\omega)\right)=\left\langle\left[f_{i}^{\dagger}, f_{i}\right]_{+}\right\rangle=1
$$

for the spectral functions in Figs. 6 and 7 . We found that for the two small $U$ values in Fig. [6 the sum rule was perfectly fulfilled whereas for the spectral functions with $U=2.0$ and 3.0 (Fig. 6b and Fig. 6 6 ) the agreement was less satisfactory. For instance, for $U=3.0$ a value of 0.9 instead of 1 was found. The origin of these deviations can be understood as follows. First, from Eqs. (40) and (41) one finds that (51) is fulfilled if

$$
\left|\nu_{\lambda}\right|^{2}+\frac{1}{N^{2}} \sum_{k k^{\prime}}\left|\mu_{k k^{\prime}, \lambda}\right|^{2} f\left(\varepsilon_{k, \lambda}\right)\left(1-f\left(\varepsilon_{k^{\prime}, \lambda}\right)\right)=1
$$

is satisfied for all values of $\lambda$. For the original model relation (52) is trivially valid. By restricting oneself to the lowest order processes in the renormalization equations for the 
$\lambda$-dependent coefficients in (39) one can show that the sum rule is fulfilled for $(\lambda-\Delta \lambda)$ if it was fulfilled for $\lambda$. However, there are deviations due to higher order renormalization contributions for larger $U$ and small values of $\lambda$ as follows from expression (25) for $A_{k, k^{\prime}}^{\lambda, \Delta \lambda}$. Also important for the deviations is the chosen simple operator structure of the generator $X_{\lambda, \Delta \lambda}$ from (12). Note however that higher order processes in $U$ are suppressed for larger system sizes $N$. Thus, in order to control the influence of higher order terms a finite size scaling in $N$ should be done. Instead, in the present approach a simpler truncation procedure has been used. The renormalization was stopped at the smallest $\lambda$ value at which the influence of higher order terms was still negligible. This leads to the observed difference in the sum rule as compared to the exact result.
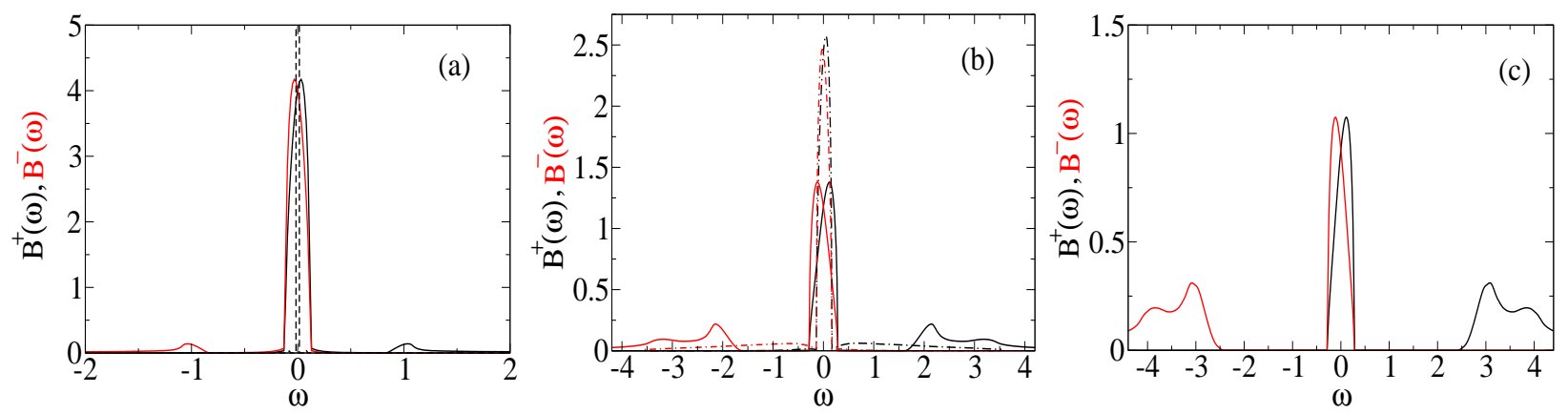

FIG. 6: (a) One-particle spectral functions $B^{+}(\omega)$ (black) and $B^{-}(\omega)$ (red) of $f$-electrons as function of $\omega$ for $U=0.1$ (dashed line) and $U=1.0$ (solid line), and $n_{f}=n_{c}=1 / 2, T=0.1$. For both $U$ values the coherent excitation dominates the spectrum. (b),(c) Same quantity as in Fig. 6a for two larger values of $U, U=2.0$ (b) and $U=3.0$ (c) and $T=0.1$ (solid lines). Note that now the incoherent excitations have gained considerable weight as compared to the case of panel (a). For $U=2.0$ (b) the spectral function is also shown for a higher temperature $T=0.2$ (dashed dotted line).

\section{CONCLUSIONS}

In summary, in this paper we have applied a recently developed projector-based renormalization method (PRM) to the spinless Falicov-Kimball model in one dimension and at 

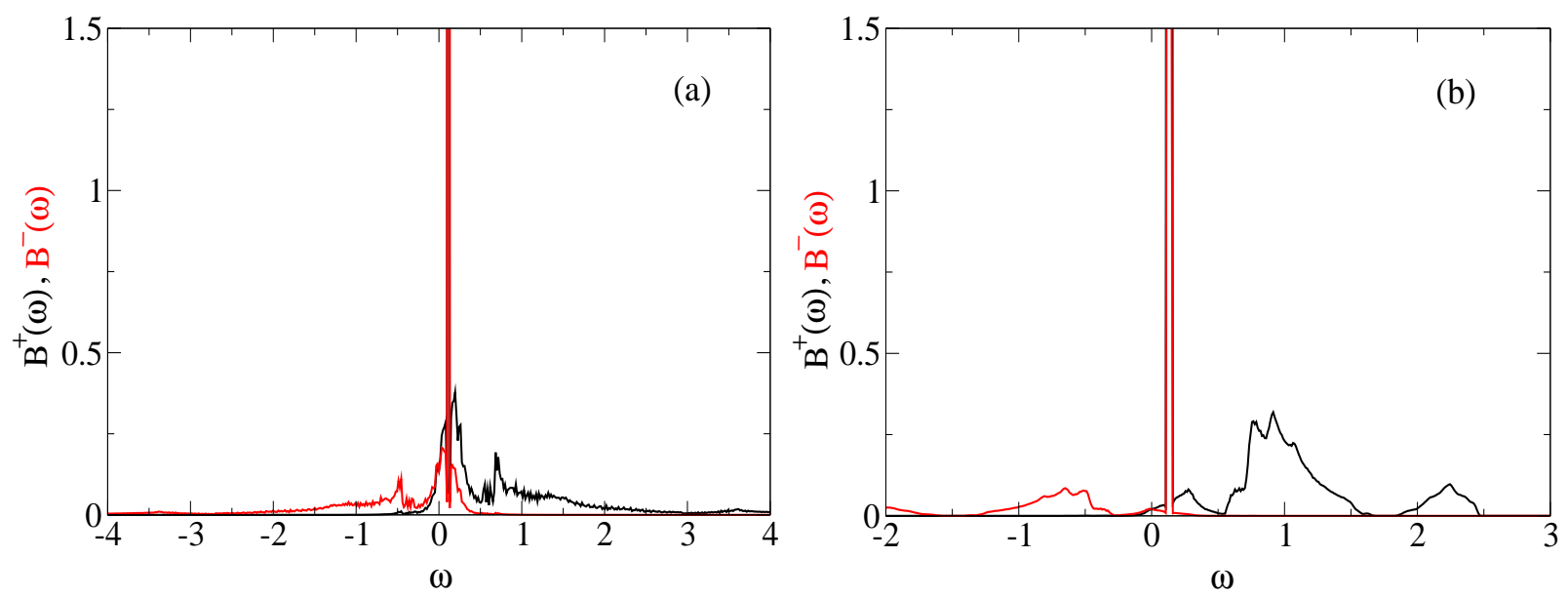

FIG. 7: Same quantity as in Fig. [6 for $U=1.0$ (a) and $U=2.0$ (b) and $n_{f}=1 / 3$.

half-filling $n_{f}+n_{c}=1$. As a starting point in the formalism, we have taken the one-particle part of the initial Hamiltonian (11) as unperturbed Hamiltonian $\mathcal{H}_{0}$ and the local Coulomb interaction $U$ between conduction and localized electrons as interaction $\mathcal{H}_{1}$. The elimination of the latter part leads to the renormalization of the Hamiltonian. Thereby a new densitydensity interaction between the localized electrons is generated. Finally, a number of static and dynamic quantities has been calculated. Note that the present formuation of the PRM is a weak-coupling approach which however should lead to reliable results for $U$-values up to the order of $t$. It is shown that the investigation of the small temperature behavior enables us also to extract valuable information about the zero temperature properties of static and dynamic quantities.

\section{Acknowledgments}

We would like to acknowledge fruitful discussions with J. Freericks, Ch. Geibel and A. Hübsch. This work was supported by the DFG through the research program SFB 463, and by DFG grant No: Dr 274/10-1. One of us (VZ) would like to acknowledge the support by the National Science Foundation under grant number DMR-0210717. 


\section{APPENDIX A: OPERATOR TRANSFORMATION OF $\mathcal{H}_{\lambda}$}

In this appendix we evaluate the renormalized Falicov-Kimball Hamiltonian obtained by the unitary transformation $\mathcal{H}_{(\lambda-\Delta \lambda)}=\exp \left\{X_{\lambda, \Delta \lambda}\right\} \mathcal{H}_{\lambda} \exp \left\{-X_{\lambda, \Delta \lambda}\right\}$, where $X_{\lambda, \Delta \lambda}$ is given by (18) The transformed Hamiltonian $\mathcal{H}_{\lambda-\Delta \lambda}$ has no excitations with transfer energies $\left|\varepsilon_{k, \lambda-\Delta \lambda}-\varepsilon_{k^{\prime}, \lambda-\Delta \lambda}\right|>\lambda-\Delta \lambda$. Let us first consider the transformation of $\mathcal{H}_{0, \lambda}$

$$
e^{X_{\lambda, \Delta \lambda}} \mathcal{H}_{0, \lambda} e^{-X_{\lambda, \Delta \lambda}}-\mathcal{H}_{0, \lambda}=\sum_{k} \varepsilon_{k, \lambda} \sum_{n=1}^{\infty} \frac{1}{n !} \mathbf{X}_{\lambda, \Delta \lambda}^{n} c_{k}^{\dagger} c_{k}
$$

where we have introduced a new superoperator $\mathbf{X}_{\lambda, \Delta \lambda}$ which is defined by the commutator of the generator $X_{\lambda, \Delta \lambda}$ with operators $\mathcal{A}$ on which $\mathbf{X}_{\lambda, \Delta \lambda}$ is applied, $\mathbf{X}_{\lambda, \Delta \lambda} \mathcal{A}=\left[X_{\lambda, \Delta \lambda}, \mathcal{A}\right]$. Note that the operators $c_{k}^{\dagger} c_{k}$ are the only part of $\mathcal{H}_{0, \lambda}$ which do not commute with $X_{\lambda, \Delta \lambda}$. We successively evaluate the commutators on the right-hand side of (A1). By use of

$$
\mathbf{X}_{\lambda, \Delta \lambda} c_{k}^{\dagger} c_{k}=-\frac{1}{N} \sum_{\tilde{k}, i} A_{k \tilde{k}} \Theta_{k \tilde{k}}^{\lambda, \Delta \lambda}\left(e^{i(\tilde{k}-k) R_{i}} \delta n_{i} c_{\tilde{k}}^{\dagger} c_{k}+e^{i(k-\tilde{k}) R_{i}} \delta n_{i} c_{k}^{\dagger} c_{\tilde{k}}\right)
$$

we first obtain

$$
\mathbf{X}_{\lambda, \Delta \lambda} \mathcal{H}_{0, \lambda}=\frac{1}{N} \sum_{k k^{\prime} i} A_{k k^{\prime}} \Theta_{k k^{\prime}}^{\lambda . \Delta \lambda}\left(\varepsilon_{k^{\prime}, \lambda}-\varepsilon_{k, \lambda}\right) e^{i\left(k-k^{\prime}\right) R_{i}} \delta n_{i} c_{k}^{\dagger} c_{k^{\prime}}
$$

Next by applying $\mathbf{X}_{\lambda, \Delta \lambda}$ on $\mathbf{X}_{\lambda, \Delta \lambda} \mathcal{H}_{0, \lambda}$ where products of two fermions and of two local density operators $\delta n_{j} \delta n_{i}$ occur. In order to keep only operators which are also present in $\mathcal{H}_{(\lambda-\Delta \lambda)}$ a factorization approximation has to be performed. With

$$
\begin{aligned}
& \mathbf{X}_{\lambda, \Delta \lambda} \delta n_{i} c_{k}^{\dagger} c_{k^{\prime}}= \frac{1}{N} \sum_{\tilde{k}, j} A_{\tilde{k} k} \Theta_{\tilde{k} k}^{\lambda, \Delta \lambda} e^{i(\tilde{k}-k) R_{j}}\left(\left\langle\delta n_{i} \delta n_{j}\right\rangle c_{\tilde{k}}^{\dagger} c_{k^{\prime}}\right. \\
&\left.+\delta_{k^{\prime}, \tilde{k}} \delta n_{i} \delta n_{j}\left\langle c_{k^{\prime}}^{\dagger} c_{k^{\prime}}\right\rangle-\delta_{k^{\prime}, \tilde{k}}\left\langle\delta n_{i} \delta n_{j}\right\rangle\left\langle c_{\tilde{k}}^{\dagger} c_{k^{\prime}}\right\rangle\right) \\
&- \frac{1}{N} \sum_{\tilde{k}, j} A_{\tilde{k} k^{\prime}} \Theta_{\tilde{k} k^{\prime}}^{\lambda, \Delta \lambda} e^{-i\left(\tilde{k}-k^{\prime}\right) R_{j}}\left(\left\langle\delta n_{i} \delta n_{j}\right\rangle c_{k}^{\dagger} c_{\tilde{k}}\right. \\
&\left.+\delta_{k, \tilde{k}} \delta n_{i} \delta n_{j}\left\langle c_{k}^{\dagger} c_{k}\right\rangle-\delta_{k, \tilde{k}}\left\langle\delta n_{i} \delta n_{j}\right\rangle\left\langle c_{k}^{\dagger} c_{\tilde{k}}\right\rangle\right)
\end{aligned}
$$

one finds

$$
\begin{aligned}
\mathbf{X}_{\lambda, \Delta \lambda}^{2} \mathcal{H}_{0, \lambda}= & \frac{2}{N^{2}} \sum_{k k^{\prime} i j} A_{k k^{\prime}}^{2} \Theta_{k k^{\prime}}^{\lambda, \Delta \lambda}\left(\varepsilon_{k^{\prime}, \lambda}-\varepsilon_{k, \lambda}\right) \cos \left[i\left(k^{\prime}-k\right)\left(R_{i}-R_{j}\right)\right] \\
& \times\left(\left\langle\delta n_{i} \delta n_{j}\right\rangle c_{\tilde{k}}^{\dagger} c_{k^{\prime}}+\delta_{k^{\prime}, \tilde{k}} \delta n_{i} \delta n_{j}\left\langle c_{k^{\prime}}^{\dagger} c_{k^{\prime}}\right\rangle-\delta_{k^{\prime}, \tilde{k}}\left\langle\delta n_{i} \delta n_{j}\right\rangle\left\langle c_{\tilde{k}}^{\dagger} c_{k^{\prime}}\right\rangle\right)
\end{aligned}
$$


or by using translational invariance

$$
\begin{aligned}
\mathbf{X}_{\lambda, \Delta \lambda}^{2} \mathcal{H}_{0, \lambda} & =\frac{2}{N} \sum_{k k^{\prime}} A_{k k^{\prime}}^{2} \Theta_{k k^{\prime}}^{\lambda, \Delta \lambda}\left(\varepsilon_{k^{\prime}, \lambda}-\varepsilon_{k, \lambda}\right) \\
& \times\left(C_{\rho}\left(k^{\prime}-k\right) c_{k}^{\dagger} c_{k}+\left\langle c_{k}^{\dagger} c_{k}\right\rangle \frac{1}{N} \sum_{i j} \delta n_{i} \delta n_{j} e^{i\left(k-k^{\prime}\right)\left(R_{i}-R_{j}\right)}-\left\langle c_{k}^{\dagger} c_{k}\right\rangle C_{\rho}\left(k^{\prime}-k\right)\right)
\end{aligned}
$$

Note that in deriving (A5) and (A6) it was assumed that the number of $k$ points which are integrated out in the step from $\lambda$ to $(\lambda-\Delta \Lambda)$ is small compared to the total number of $k$ points. Therefore, the product of two product $\delta$ functions $\Theta_{k} k^{\prime}$ and $\Theta_{\tilde{k} \tilde{k}^{\prime}}$ is only nonzero when the wavevectors are pairwise equal to each other (shell condition). In the next step, $\mathbf{X}_{\lambda, \Delta \lambda}$ is again applied on $c_{k}^{\dagger} c_{k}$. Thus by use of (A2) one finds

$$
\mathbf{X}_{\lambda, \Delta \lambda}^{3} \mathcal{H}_{0, \lambda}=\frac{1}{N} \sum_{k k^{\prime} i}\left[-\frac{2^{2} C_{\rho}\left(k-k^{\prime}\right) A_{k k^{\prime}}^{2}}{N}\right] A_{k k^{\prime}} \Theta_{k k^{\prime}}^{\lambda, \Delta \lambda}\left(\varepsilon_{k^{\prime}, \lambda}-\varepsilon_{k, \lambda}\right) e^{i\left(k-k^{\prime}\right) R_{i}} \delta n_{i} c_{k}^{\dagger} c_{k^{\prime}}
$$

which has again the structure of $\mathbf{X}_{\lambda, \Delta \lambda} \mathcal{H}_{0, \lambda}$ except of the additional prefactor in the bracket [...]. thus one may sum up all commutator terms from (A1) in order to obtain a compact expression for (A1). In the same way one may proceed with the transformation of $\mathcal{H}_{1, \lambda}$.

\section{APPENDIX B: GREEN FUNCTION $\mathcal{G}_{0}(\omega)$ OF THE LATTICE GAS MODEL}

To evaluate the Green function $\mathcal{G}_{0}(\omega)$

$$
\mathcal{G}_{0}(\omega)=i \int_{0}^{\infty} d t e^{i(\omega+i \eta) t}\left\langle\left[f_{i}(t), f_{i}^{\dagger}\right]_{+}\right\rangle_{\tilde{\mathcal{H}}_{f}}
$$

we best use the Mori-Zwanzig projection formalism. First, let us define an anticommutator scalar product for operators $\mathcal{A}, \mathcal{B}$ by $(\mathcal{A} \mid \mathcal{B})=\left\langle\left[\mathcal{A}^{\dagger}, \mathcal{B}\right]_{+}\right\rangle_{\tilde{\mathcal{H}}_{f}}$. In addition, we introduce the Liouville operator $\tilde{\mathbf{L}}_{f}$ belonging to the lattice gas Hamiltonian $\tilde{\mathcal{H}}_{f}$. It acts on any operator $\mathcal{A}$ of the unitary space as $\tilde{\mathbf{L}}_{f} \mathcal{A}=\left[\mathcal{H}_{f}, \mathcal{A}\right]$. Thus $(\underline{\mathrm{B} 1})$ can be rewritten as

$$
\mathcal{G}_{0}(\omega)=i \int_{0}^{\infty} d t e^{i(\omega+i \eta) t}\left(f_{i}^{\dagger} \mid e^{-i \tilde{\mathbf{L}}_{f} t} f_{i}^{\dagger}\right)=\left(f_{i}^{\dagger} \mid \frac{1}{\tilde{\mathbf{L}}_{f}-\omega-i \eta} f_{i}^{\dagger}\right)
$$

The well-known operator identity of the projection formalism ${ }^{20}$ can be used to transform $\mathcal{G}_{0}(\omega)$ into

$$
\mathcal{G}_{0}(\omega)=\frac{1}{\Omega-\omega-\Sigma(\omega)}
$$


where the frequency term $\Omega$ and the selfenergy $\Sigma(\omega)$ are given by

$$
\Omega=\left(f_{i}^{\dagger} \mid \tilde{\mathbf{L}}_{f} f_{i}^{\dagger}\right) \quad \Sigma(\omega)=\left(\tilde{\mathbf{L}}_{f} f_{i}^{\dagger} \mid q \frac{1}{\mathbf{Q} \tilde{\mathbf{L}}_{f} \mathbf{Q}-\omega-i \eta} \mathbf{Q} \tilde{\mathbf{L}}_{f} f_{i}^{\dagger}\right)
$$

Note that in ( $(\underline{\mathrm{B} 3})$ and $(\underline{\mathrm{B} 4})$ we have already used that $\left(f_{i}^{\dagger} \mid f_{i}^{\dagger}\right)=\left\langle\left[f_{i}, f_{i}^{\dagger}\right]_{+}\right\rangle_{\tilde{\mathcal{H}}_{f}}=1$. The quantity $\mathbf{Q}$ is like $\mathbf{L}_{f}$ a superoperator which acts on usual operators of the unitary space. $\mathbf{Q}$ is a projection operator and projects on the subspace of the Liouville space perpendicular to $\left.\mid f_{i}^{\dagger}\right)$, i.e., it is defined by $\left.\left.\left.\mathrm{Q} \mid \mathcal{A}\right)=\mid \mathcal{A}\right)-\mid f_{i}^{\dagger}\right)\left(f_{i}^{\dagger} \mid \mathcal{A}\right)$. To evaluate $\Omega$ and $\Sigma(\omega)$ we need

$$
\left.\left.\left.\left.\left.\tilde{\mathbf{L}}_{f} \mid f_{i}^{\dagger}\right)=\tilde{\varepsilon}_{f} \mid f_{i}^{\dagger}\right)+2 \sum_{j(\neq i)} \tilde{g}_{i j} \mid \delta n_{j} f_{i}^{\dagger}\right) \quad \text { and } \quad \mathbf{Q} \tilde{\mathbf{L}}_{f} \mid f_{i}^{\dagger}\right)=2 \sum_{j(\neq i)} \tilde{g}_{i j} \mathbf{Q} \mid \delta n_{j} f_{i}^{\dagger}\right)
$$

where in the second relation the first term of $\left.\tilde{\mathbf{L}}_{f} \mid f_{i}^{\dagger}\right)$ drops due to the presence of $\mathbf{Q}$. As is easily seen, we obtain $\Omega=\tilde{\varepsilon}_{f}$ and

$$
\Sigma(\omega)=4 \sum_{j(\neq i)} \sum_{l(\neq i)} \tilde{g}_{i l}^{*} \tilde{g}_{i j}\left(\delta n_{l} f_{i}^{\dagger}\left|\mathbf{Q} \frac{1}{\mathbf{Q} \tilde{\mathbf{L}}_{f} \mathbf{Q}-\omega-i \eta} \mathbf{Q}\right| \delta n_{j} f_{i}^{\dagger}\right)
$$

Note that the local density operators $\delta n_{l}$ and $\delta n_{j}$ in the bra- and ket vector of (Bי $)$ commute with $\tilde{\mathcal{H}}_{f}$. Therefore, we can approximate $\Sigma(\omega)$ by an expression proportional to $\mathcal{G}_{0}$

$$
\Sigma(\omega)=\kappa^{2} \mathcal{G}_{0}(\omega) \quad \kappa^{2}=4 \sum_{j(\neq i)} \sum_{l(\neq i)} \tilde{g}_{i l}^{*} \tilde{g}_{i j}\left\langle\delta n_{l} \delta n_{j}\right\rangle_{\tilde{\mathcal{H}}_{f}}
$$

which is identical to (42). Note that $\kappa^{2}$ in (B7) is independent of the lattice site $i . \Sigma(\omega)$ together with (B3) forms a quadratic equation for $\mathcal{G}_{0}(\omega)$ which can be solved. It is easily seen that the imaginary part is given by

$$
\Im \mathcal{G}_{0}(\omega)=\frac{1}{\kappa} \sqrt{1-\frac{\left(\omega-\tilde{\varepsilon}_{f}\right)^{2}}{4 \kappa^{2}}}
$$

for the frequency range $\left|\omega-\tilde{\varepsilon}_{f}\right| \leq 2 \kappa$ and $\mathcal{G}_{0}(\omega)=0$ elsewhere. Thus, $\Im \mathcal{G}_{0}(\omega)$ is nonzero only in a small frequency region around $\omega=\tilde{\varepsilon}_{f}$ of width $\pm 2 \kappa$ where $\kappa$ is determined by static density-density correlations of the lattice gas model. The maximum value of $\Im \mathcal{G}_{0}(\omega)$ is located at $\omega=\tilde{\varepsilon}_{f}$ and is given by $1 / \kappa$.

From $\Im \mathcal{G}_{o}(\omega)$ the functions $B_{0}^{+}(\omega)$ and $B_{0}^{-}(\omega)$ are determined by help of the fluctuationdissipation theorem

$$
B_{0}^{+}(\omega)=(1-f(\omega)) \frac{1}{\pi} \Im \mathcal{G}_{0}(\omega) \quad B_{0}^{-}(\omega)=f(\omega) \frac{1}{\pi} \Im \mathcal{G}_{0}(\omega)
$$


where $f(\omega)$ is again the Fermi function. From (B9) and (401), (411) the $f$-electron Green functions of the original Falicov-Kimball model can be found.

1 L. M. Falicov and J. C. Kimball, Phys. Rev. Lett. 22, 997 (1969)

2 J. K. Freericks and V. Zlatić, Phys. Rev. B 58, 322 (1998).

3 J. K. Freericks and V. Zlatic, Rev. Mod. Phys. 75, 1333 (2003).

4 U. Brandt and C. Mielsch, Z. Phys. B: Condens. Matter 75, 365 (1989).

5 P. G. J. van Dongen and D. Vollhardt, Phys. Rev. Lett. 65, 1663 (1990).

6 T. Kennedy and E. H. Lieb, Physica A 138, 320 (1986).

7 E. H. Lieb, Physica A 140, 240 (1986).

8 S. Sykora, A. Hübsch, and K. W. Becker, Eur. Phys. J. B 51, 181 (2006).

9 K. W. Becker, A. Hübsch, and T. Sommer, Phys. Rev. B 66, 235115 (2002).

10 W. Kohn, Phys. Rev. A 133, 171 (1964).

11 J. R. Schrieffer, P. A. Wolff, Phys. Rev. 149, 491 (1966).

12 P. W. Anderson, J. Phys. C: Solid State Phys. 3, 2436 (1970).

13 F. Wegner, Ann. Phys. (Leipzig) 3, 77 (1994).

14 S. D. Głazek and K.G. Wilson, Phys. Rev. D 48, 5863 (1993); S. D. Głazek and K.G. Wilson, Phys. Rev. D 49, 4214 (1994).

15 H. R. Ott and Z. Fisk, in Handbook on the Physics and Chemistry of the Actinides, edited by A: J. Freeman and G. H. Lander (Elsevier, Amsterdam, 1987).

16 P. Farkasovsky, Phys. Rev. B 54, 7865 (1996).

17 P. Farkasovsky, Int. J. Mod. Phys. B 17, 4897 (2003).

18 in N. Nagaosa, Quantum Field Theory in Strongly Correlated Electronic Systems Springer-Verlag Berlin Heidelberg New York, 1998.

19 M. M. Maska and K. Czajka, Phys. Rev. B 74, 035109 (2006).

20 in The Quantum Statistics of Dynamic Processes, E. Fick and G. Sauermann, Springer series in Solid-State Sciences 86, 1990.

21 R. Lyzwa, Physica A 192, 231 (1993). 